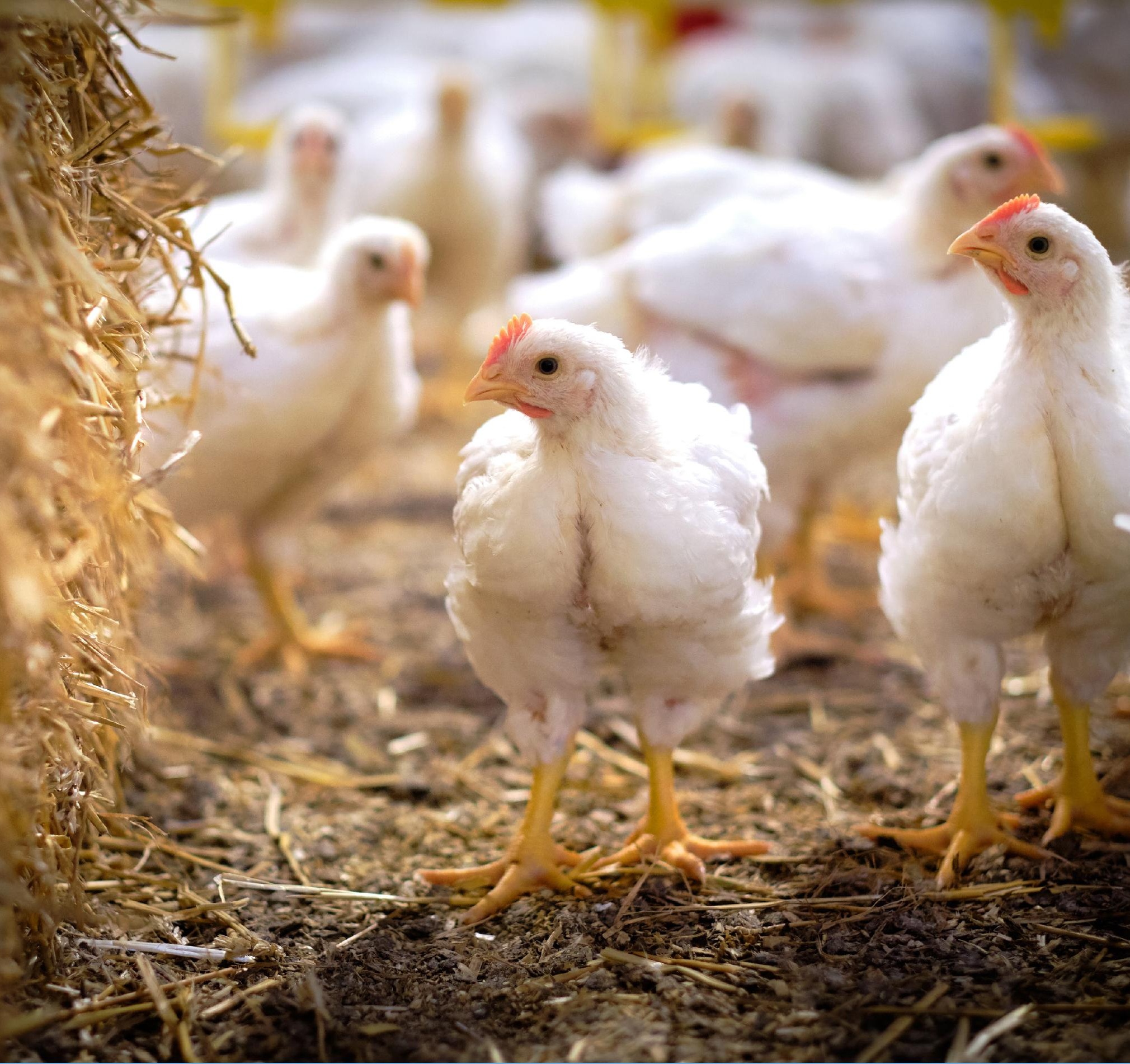

Literature update on effective environmental enrichment and light provision in broiler chickens

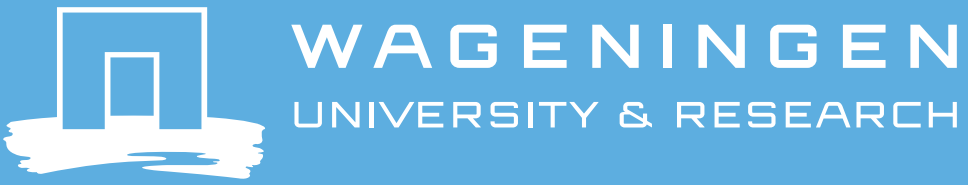





\section{Literature update on effective environmental enrichment and light provision in broiler chickens}

C. Souza da Silva and I.C. de Jong

Wageningen Livestock Research

This research was conducted by Wageningen Livestock Research, commissioned and funded by the Ministry of Agriculture, Nature and Food Quality, Hubbard Breeders, Kuikenbroederij Morren, De Hoop Mengvoeders, HATO BV, 2SistersStorteboom, Meyn Food Processing Technology BV, MSD Animal Health, Petersime, Avined, adVee Dierenartsen, Dierenbescherming, and Food and Agricultural Initiative within the framework of the public-private partnership 'Trager groeiende vleeskuikens: op weg naar integraal duurzaam dierenwelzijn' (TKI-AF-18091, BO47-001-052)

Wageningen Livestock Research

Wageningen, September 2019

Report 1204 
Souza da Silva, C., de Jong, I.C., 2019. Literature update on effective environmental enrichment and light provision in broiler chickens. Wageningen Livestock Research, Report 1204.

\section{Summary}

The results of a literature study to the effect of different types of environmental enrichment and light conditions on broiler chickens welfare are described, in order to attempt to provide an environment to slow-growing broiler chickens that better meets their behavioural requirements. With respect to environmental enrichment, a review paper has been used as a starting point and more recent information has been collected and summarised. With respect to lighting, in consultation with stakeholders we chose to limit the literature study to a few potential interesting areas of research (e.g. natural light provision and its variation across the broiler house). There are several research questions in relation to enrichment provision, e.g., optimal perch design, multiple use of enrichments and the actual number of enrichments that should be provided. Currently, little is known about the need for light in slow-growing broiler chickens and how this interacts with the environmental enrichment offered. Future research priorities include the optimization of methods of natural light provision (which is often applied in higher welfare indoor systems with slow-growing breeds), testing effects of ultraviolet wavelengths on chicken behaviour, and light colour preferences in slow-growing breeds.

This report can be downloaded for free at https://doi.org/10.18174/504630 or at www.wur.nl/livestock-research (under Wageningen Livestock Research publications).

\section{(C) 2019 Wageningen Livestock Research}

P.O. Box 338, 6700 AH Wageningen, The Netherlands, T +31 (0)317 483953 ,

E info.livestockresearch@wur.nl, www.wur.nl/livestock-research. Wageningen Livestock Research is part of Wageningen University \& Research.

All rights reserved. No part of this publication may be reproduced and/or made public, whether by print, photocopy, microfilm or any other means, without the prior permission of the publisher or author.

Wageningen Livestock Research is NEN-EN-ISO 9001:2015 certified.

All our research commissions are in line with the Terms and Conditions of the Animal Sciences Group. These are filed with the District Court of Zwolle. 


\section{Table of contents}

$\begin{array}{ll}\text { Foreword } & 5\end{array}$

$\begin{array}{ll}\text { Summary } & 7\end{array}$

1

$\begin{array}{ll}\text { Introduction } & 9\end{array}$

2

$\begin{array}{ll}\text { Materials and methods } & 11\end{array}$

$2.1 \quad$ Literature study environmental enrichment 11

$\begin{array}{ll}2.2 & \text { Literature study light provision } \\ 2.3 & 11\end{array}$

$\begin{array}{lll}2.3 & \text { Practical inventory } & 11\end{array}$

3

$\begin{array}{ll}\text { Results on Environmental Enrichment } & 12\end{array}$

$3.1 \quad$ Literature study $\quad 12$

3.1.1 Point-Source Objects in Conventional Environments 12

3.1.2 More Complex Enriched Environments with Outdoor Access 26

3.1.3 More Complex Enriched Environments: The Higher Welfare Indoor
Systems

3.2 Practical inventory 26

3.2.1 Overview of the requirements for the use of enrichment in the different broiler production systems in the Netherlands and United Kingdom 26

3.2.2 List of enrichments that are commonly applied into practice in the different broiler production systems in the Netherlands

4.1 Literature study

4.2 Practical inventory

5.1 Literature study

5.1.1 Effects of light during incubation $\quad 31$

5.1.2 Effects of ultraviolet wavelengths on chicken behaviour 31

5.1.3 Light colour preferences of broiler chickens 32

$\begin{array}{lll}6.1 & \text { Environmental enrichment } & 35\end{array}$

$\begin{array}{lll}6.2 & \text { Lighting conditions } & 36\end{array}$

$\begin{array}{lll}6.3 & \text { Recommendations for future research } & 37\end{array}$

$\begin{array}{ll}\text { References } & 38\end{array}$

$\begin{array}{ll}\text { Appendix } 1 & 43\end{array}$ 



\section{Foreword}

The project 'Trager groeiende vleeskuikens: Op weg naar integraal duurzaam dierenwelzijn' (Slow growing broiler chickens: steps forward to an integral sustainable animal welfare) is a public-private partnership between the Ministry of Agriculture, Nature and Food Quality, a consortium of various private parties and organizations within the slow growing broiler production chain and Wageningen Livestock Research. The project aims to contribute to a broader sustainable and healthy slow growing broiler chain in the Netherlands.

This report contains the results of a literature review and practical inventory of the available information about slow growing broilers in relation to their rearing environment, in order to meet their behavioural needs.

For the current study, scientists of Wageningen Livestock Research worked together with representatives from the consortium, and the authors thank the private partners of the project team for their worthwhile input.

Dr. R.A. (Rick) van Emous, project leader. 


\section{Summary}

The ultimate goal of the project 'PPS Trager groeiende vleeskuikens: Op weg naar integraal duurzaam dierenwelzijn' (Slow growing broiler chickens: steps forward to an integral sustainable animal welfare) is to offer an environment to slow-growing broiler chickens which meets their behavioural needs. As a starting point to define research questions specifically targeted to optimise housing conditions of these chickens, the current report was written to summarise the state-of-the art of knowledge with respect to effective environmental enrichment and lighting conditions used for slow-growing broiler chickens at the moment. With respect to environmental enrichment, the review paper of Riber and collaborators (2018) has been used as a starting point and more recent information has been collected and summarised. With respect to lighting, after consultation with stakeholders, the literature study has been narrowed to three potential interesting areas of research: (1) effects of natural light provision and its variation across the broiler house; (2) effects of ultraviolet wavelengths on chicken behaviour; and (3) light colour preferences of broiler chickens.

Elevated resting places (such as perches and platforms) are generally accepted as an effective form of enrichment for broiler chickens. Perch use varies, whereas platforms are often well used by slowgrowing broiler chickens. Evaluation of optimal designs of perches should be considered in order to stimulate perch use, but the risk for increased prevalence of breast blisters and keel bone damage with perches should be also considered in slow-growing broiler chickens. Substrate bales are considered as a type of enrichment with multiple functions that have not been fully explored in broiler production systems yet. Birds may use bales for perching, for clustering around it (protection), and for explorative behaviour (pecking). To date, no studies have specifically documented the multiple uses of one enrichment. In addition, there are no scientifically proved indications of an optimum number of enrichments (such as bales and elevated resting places) per number of chickens that should be provided. Therefore, studies on multi-functional types of enrichments and on the optimum number of enrichments that should be provided in accordance to group size are highly recommended.

Currently, little is known about the need for light in slow-growing broiler chickens and how this interacts with the environmental enrichment offered. There are various potential interesting areas of research with regard to light, and some of these have been mentioned in the present report. One important issue regards the provision of natural light in the so-called higher welfare indoor systems used for slow-growing broiler chicken production in the Netherlands. It is unclear whether the methods of providing natural light really meet the need of all birds at all ages in these systems. There is also an important question about variation in natural light intensity across the broiler house that may stimulate birds to use different areas around the house for different behaviours, and this merits further research as well. Optimization of the method of providing natural light is also needed, and experiments testing differences between roof and side wall windows effects on broiler behaviour and welfare are encouraged. To date, there are no studies that have been conducted in the Netherlands to test effects of ultraviolet wavelengths on chicken behaviour and to test light colour preferences in slow-growing breeds. Thus, studies on ultraviolet wavelengths and colour preferences in slow-growing broilers in relation to age and behaviour are needed.

To conclude, in relation to the current lack of knowledge on the behavioural requirements of slowgrowing broiler chickens, various questions were raised during the writing and discussion of the present report. In the next phase of the project in 2020 and beyond, a limited number of research questions will be selected and used for the design of experiments on enrichment provision and on light provision in slow-growing broiler chickens. Different slow-growing breeds are used nowadays, and may differ with regard to their requirements for enrichment and light. Nevertheless, the main focus of the experiments will be on Hubbard slow-growing breeds which are considered representative of the Dutch slow-growing broiler market at the moment. 


\section{Introduction}

In the Netherlands, the production of broiler chickens experienced a significant change between 2014 and 2016. This was due to the rapid introduction to all Dutch supermarkets of their own 'Kip van Morgen' (Chicken of tomorrow) concept (Saatkamp, et al., 2019) in addition to the already existing 'Beter Leven' (Better life) concept that was introduced by the Dutch Society for the Protection of Animals (SPA) in 2007. Both alternative production concepts are aimed at improving broiler welfare. As a result, currently around $35-40 \%$ of broiler chickens produced in the Netherlands are kept according to a slower-growing concept (Avined, 2019; Van Boekholt, pers. comm.). Slow-growing broiler chickens are defined as broiler chickens produced by slow-growing female parent stock that are crossed with a regular or slow-growing male. These broiler breeds are either accredited by the Dutch SPA with the 'Beter Leven' quality label (maximum average daily growth of 45 grams) or fall within the standards of the original 'Kip van Morgen' concept (maximum average daily growth of 50 grams) (Ellen, et al., 2012; Saatkamp, et al., 2019). Based on the number of broiler breeders present in the Netherlands, it is estimated that the Hubbard breeds have a share of approximately $75 \%$ of the total slow-growing broiler market (Van Boekholt, pers. comm.), and are considered representative of the Dutch slow-growing broiler market at the moment.

With regard to animal welfare, it is important that farm animals are kept in an environment that meets their behavioural needs. The possibilities to perform natural behaviour are an essential part of animal welfare and contribute to the animal's experiencing positive emotions (Welfare Quality, 2009; Boissy, et al., 2007; Fraser, 1995). Chickens are day-active animals that spend a large part of the light period gathering food and being active (Dawkins, 1989). Also, chickens are intelligent animals that need cognitive challenges and actively collect information from their environment (Marino, 2017). The visual spectrum of the chicken is larger than that of humans, and the type of light that is provided determines how a chicken experiences the environment (Prescott and Wathes, 1999). Therefore, a greater variety in environmental enrichment and light, than is currently applied in commercial broiler houses, is expected to better meet the behavioural needs of broiler chickens. However, further research is still needed, particularly in recently introduced slow-growing breeds.

Effective environmental enrichment stimulates natural behaviour, can reduce negative feelings such as anxiety, and contributes to experiencing positive emotions (Riber, et al., 2018) and thus potentially contributes to broiler chickens' welfare. Although much research is currently being done on effective environmental enrichment for broiler chickens, the majority of previous and current research focused on regular, fast-growing breeds (Bailie, et al., 2013; Bailie, et al., 2018a; Bailie, et al., 2018b; Bailie and O'Connell, 2014; Baxter, et al., 2018b; Baxter, et al., 2019; De Jong and Gunnink, 2019; Riber, et al., 2018). A one-to-one translation of effects of environmental enrichment on behaviour and other welfare indicators of fast-growing broiler chickens into slow-growing breeds is not possible, because the behavioural needs of slow-growing breeds may differ, and they are usually much more active and have a different time budget than fast-growing broiler chickens (Bokkers and Koene, 2003; Rothschild, et al., 2019). Slow-growing broiler chickens are less affected by their body weight, body shape and locomotion problems than fast-growing broiler chickens when conducting behaviour, especially in the last weeks prior to slaughter (EFSA, 2010). Thus, both the need for specific environmental enrichment as well as the effects of environmental enrichment on behaviour and other welfare indicators may differ between fast and slow-growing broiler chickens.

Currently, little is known about the need for light in broiler chickens - both in terms of intensity and spectrum, and how this interacts with the environmental enrichment offered. Broilers may, for instance, have preferences to perform specific behaviours under different light and enrichment conditions. This applies to both fast- and slow-growing broiler chickens, but the few research that has been conducted has been done on fast-growing chickens (Archer, 2018; Huth and Archer, 2015; Riber, 2015). Light intensity and wavelengths provided in current housing systems for broiler chickens, including the provision of natural light (daylight), are usually based on perceptions or knowledge from laying hens or fast-growing broiler chickens. More insight in the specific requirements 
of slow-growing broiler chickens with respect to light helps to provide housing conditions that better meet the needs of the birds, and thus contribute to broiler chickens' welfare.

In the current report, we aim to summarise the state-of-the art of knowledge with respect to effective environmental enrichment and lighting conditions for broiler chickens, as a starting point to define research questions specifically targeted to optimise housing conditions for slow-growing broiler chickens. With respect to environmental enrichment, the publication of Riber and collaborators (Riber, et al., 2018) has been used as a starting point and more recent information has been collected and summarised. With respect to lighting, in consultation with stakeholders we chose to limit the literature study to three potential interesting areas of research: (1) effects of natural light provision and its variation across the broiler house; (2) effects of ultraviolet wavelengths (UV) on chicken behaviour; and (3) light colour preferences of broiler chickens. The reasoning behind these choices is that currently many housing systems for slow-growing broiler breeds include the provision of natural light, but it is unclear whether the methods of providing natural light really meet the need of all birds at all ages. There is also an important question about variation in natural light provision across the broiler house. For example, in UK houses light is often provided in windows along the side of the house. This means that there is variation in light intensity across the house, so that birds can utilise different areas around the house for different behaviours. Broiler chickens prefer low light intensities for resting and high light intensities for active behaviours (Blatchford, et al., 2012; Rault, et al., 2017). Currently, roof windows (without shutters) provided in broiler houses do not always allow for much variation, which means that light distribution is quite uniform. Thus, individual birds do not always have the opportunity to choose to perform different behaviours at different areas (Rayner, pers. comm.). With respect to $U V_{A}$, this affects the bird's perception of the environment and may have positive effects on behaviour and welfare (Niekerk, et al., 2015). Moreover, with the increased use of light-emitting diode (LED) lights in poultry housing, broilers can be provided with different light colours depending on the age, time of the day and functional area in the house, but more insight is needed in the actual colour preferences of the chickens (Archer, 2018; Riber, 2015).

The final aim of the project 'PPS Trager groeiende vleeskuikens: Op weg naar integraal duurzaam dierenwelzijn' (Slow growing broiler chickens: steps forward to an integral sustainable animal welfare) is to offer an environment to slow-growing broiler chickens which meets their behavioural needs, e.g. for exploration, free-range use, dustbathing, foraging behaviour, resting behaviour, and promotes positive experiences, and, thus, limits the risk for negative emotions such as fearfulness. This to optimise their welfare in commercial systems, while at the same time being practically applicable and economically efficient. Therefore, this literature study and practical inventory form the basis for trials in 2020 and beyond. 


\section{Materials and methods}

\subsection{Literature study environmental enrichment}

The present literature study is based on the review paper from Riber and collaborators (Riber, et al., 2018), which gives an overview of the different kinds of environmental enrichment in all types of production systems for broiler chickens. The review was based on relevant scientific literature from the database "Web of Science" retrieved using the key words "broiler environmental enrichment." In addition, the review includes references identified on reference lists of papers reviewed. With the exception of a few textbook sources and scientific reports, only peer-reviewed references have been included, written in English or German. The time frame for inclusion into the review was that sources were published in or after the year 2000, up until year 2017.

For the purpose of section 4.1 (Behavioural needs) of the project PPS Trager groeiende vleeskuikens: Op weg naar integraal duurzaam dierenwelzijn (2019) - which is to gain insight into the needs of slowgrowing chickens with regard to environmental enrichment and light provision - the summary tables included in the paper of Riber and collaborators (Riber, et al., 2018) were expanded with relevant scientific literature published in or after the year 2017, up until year 2019. Most references were retrieved from the database "Web of Science" on June 3-5, 2019, using the same key words as in the paper of Riber and collaborators (Riber, et al., 2018). Moreover, two extra scientific reports have been included: one pilot-study report from Wageningen Livestock Research (De Jong and Van WijheKiezebrink, 2014) and a recently finished Master thesis report from Wageningen University and Research (Blaauw, 2019).

\subsection{Literature study light provision}

In consultation with stakeholders we chose to limit the literature study to three potential interesting areas of research: (1) effects of natural light provision and its variation across the broiler house; (2) effects of ultraviolet wavelengths on chicken behaviour; and (3) light colour preferences of broiler chickens. These topics and peer-reviewed references included in the present literature study were selected after personal communication with Kim Geurts (HATO Agricultural Lighting, Sittard, The Netherlands) and Annie Rayner (FAI Farms, Oxford, UK). Older scientific literature that has been published on the effects of light conditions (particularly light colour) on growth performance parameters of broiler chickens have not been included in the present literature study because it falls outside the scope of the study.

\subsection{Practical inventory}

In addition, a practical inventory was carried out with the help of stakeholders (Janny Hermans from AdVee Veterinary Practice, Ysselsteyn, The Netherlands and Henk-Jan Schuurman from De Hoop Mengvoeders B.V., Zelhem, The Netherlands) with regard to environmental enrichment and natural light provision, yielding a list of enrichments that are applied in practice, and the methods of providing natural daylight to slow-growing broiler chickens in the Netherlands. Moreover, after an open plenary discussion of the present report with all project partners, the practical inventory sessions have been further expanded. 


\section{Results on Environmental Enrichment}

\subsection{Literature study}

\subsubsection{Point-Source Objects in Conventional Environments}

The following types of point-source objects used in conventional environments are reviewed in this section: perches and platforms; panels, barriers and bales of substrates; materials and methods stimulating foraging and dustbathing behaviour; and novel objects.

\subsubsection{Perches and Platforms}

Table 1 presents the main features of the perches and platforms used in the studies reviewed by Riber and collaborators (Riber, et al., 2018), updated with results from recent publications.

Table 1 Perch and platform design for broilers used in different studies. Adapted from Riber and collaborators (Riber, et al., 2018). Information written in bold correspond to literature published in or after the year 2017, which were added to the original table of Riber and collaborators ( $N / A=$ information not available).

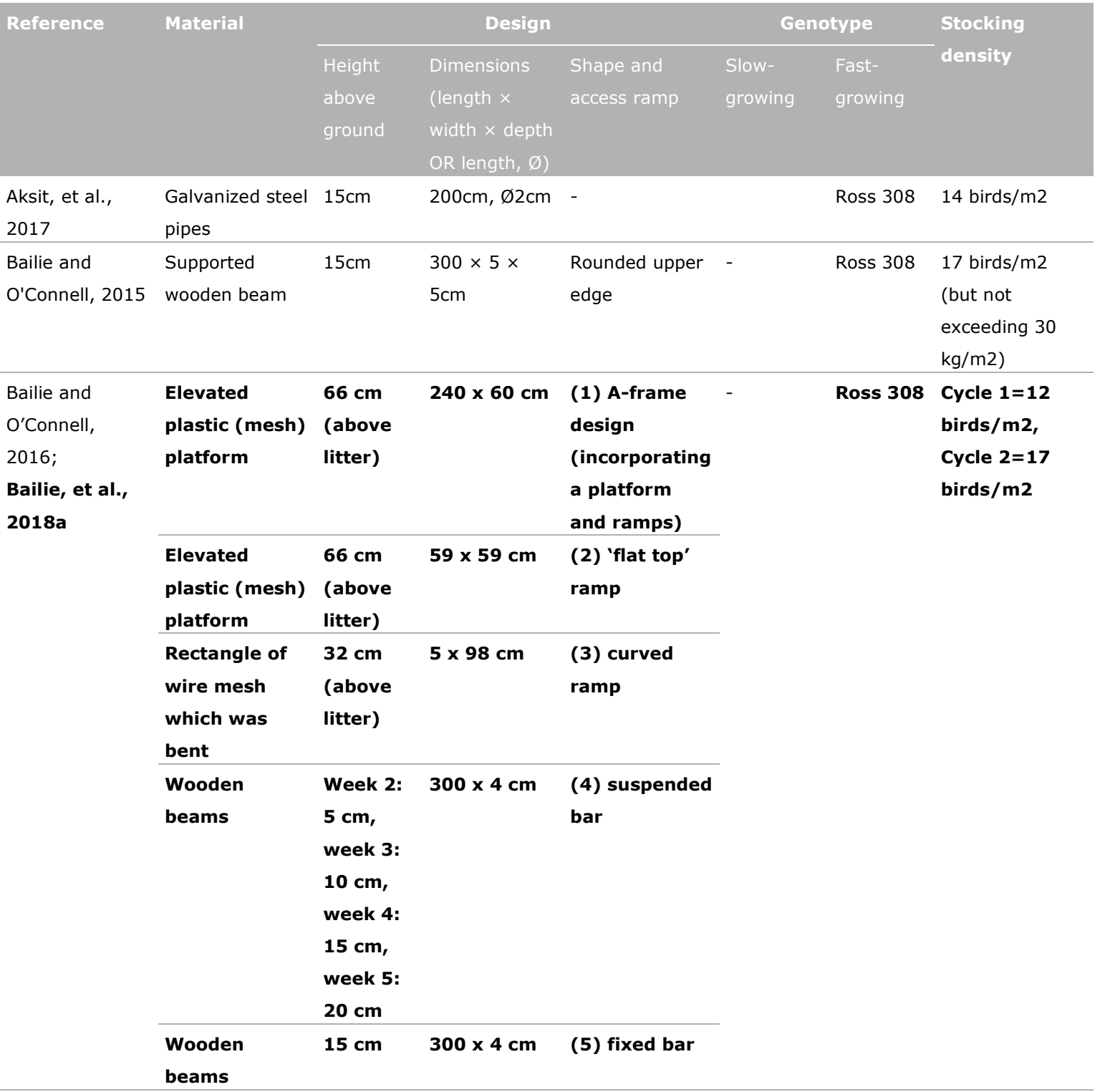




\begin{tabular}{|c|c|c|c|c|c|c|c|}
\hline \multirow[t]{3}{*}{ Reference } & \multirow[t]{2}{*}{ Material } & \multicolumn{3}{|c|}{ Design } & \multicolumn{2}{|c|}{ Genotype } & \multirow{2}{*}{$\begin{array}{l}\text { Stocking } \\
\text { density }\end{array}$} \\
\hline & & $\begin{array}{l}\text { Height } \\
\text { above } \\
\text { ground }\end{array}$ & $\begin{array}{l}\text { Dimensions } \\
\text { (length } \times \\
\text { width } \times \text { depth } \\
\text { OR length, } \varnothing \text { ) }\end{array}$ & $\begin{array}{l}\text { Shape and } \\
\text { access ramp }\end{array}$ & $\begin{array}{l}\text { Slow- } \\
\text { growing }\end{array}$ & $\begin{array}{l}\text { Fast- } \\
\text { growing }\end{array}$ & \\
\hline & $\begin{array}{l}\text { Elevated } \\
\text { plastic (mesh) } \\
\text { platform }\end{array}$ & $\begin{array}{l}\text { Week 2: } \\
5 \mathrm{~cm} \text {, } \\
\text { week 3: } \\
10 \mathrm{~cm} \text {, } \\
\text { week 4: } \\
15 \mathrm{~cm} \text {, } \\
\text { week 5: } \\
20 \mathrm{~cm}\end{array}$ & $240 \times 60 \mathrm{~cm}$ & $\begin{array}{l}\text { (6) suspended } \\
\text { platform }\end{array}$ & & & \\
\hline $\begin{array}{l}\text { Baxter, et al., } \\
2019\end{array}$ & $\begin{array}{l}\text { Elevated } \\
\text { plastic (mesh) } \\
\text { platform }\end{array}$ & $\begin{array}{l}20 \mathrm{~cm} \\
\text { (above } \\
\text { litter) }\end{array}$ & $230 \times 90 \mathrm{~cm}$ & Without ramp & - & Ross 308 & N/A \\
\hline $\begin{array}{l}\text { Bench, et al., } \\
2017\end{array}$ & $\begin{array}{l}\text { Wooden } \\
\text { beams }\end{array}$ & $10 \mathrm{~cm}$ & $\begin{array}{l}185 \times 5 \times 10 \\
\mathrm{~cm}\end{array}$ & $\begin{array}{l}\text { Without ramp, } \\
\text { perch types: } \\
\text { I-shape and } \\
\text { X-shape }\end{array}$ & - & Ross 308 & $\begin{array}{l}13.47 \\
\text { birds/m2 }\end{array}$ \\
\hline \multirow[t]{2}{*}{$\begin{array}{l}\text { Berghout, et } \\
\text { al., } 2018\end{array}$} & $\begin{array}{l}\text { Wooden } \\
\text { beams }\end{array}$ & $\begin{array}{l}6,20 \text { and } \\
35.5 \mathrm{~cm}\end{array}$ & $\begin{array}{l}127 \times 109.5 \\
\mathrm{~cm}, \varnothing 6 \mathrm{~cm}\end{array}$ & $\begin{array}{l}\text { A-frame with } \\
\text { oval shaped } \\
\text { perches }\end{array}$ & - & Ross 308 & $\begin{array}{l}21-23 \\
\text { birds/m2 (42 } \\
\mathrm{kg} / \mathrm{m} 2)\end{array}$ \\
\hline & $\begin{array}{l}\text { Elevated } \\
\text { plastic } \\
\text { platforms }\end{array}$ & $\begin{array}{l}9.5 \text { and } \\
14 \mathrm{~cm}\end{array}$ & $36 \times 56 \mathrm{~cm}$ & $\begin{array}{l}\text { Without ramp, } \\
\text { platforms } \\
\text { created by } \\
\text { placing plastic } \\
\text { transport } \\
\text { crates upside } \\
\text { down in the } \\
\text { litter }\end{array}$ & & & \\
\hline $\begin{array}{l}\text { Bergmann, et } \\
\text { al., } 2017\end{array}$ & PVC pipes & N/A & $\begin{array}{l}103 \mathrm{~m}, \\
\varnothing ? \mathrm{~cm}\end{array}$ & $\begin{array}{l}\text { Rounded } \\
\text { upper edge, } \\
\text { coated with } \\
\text { an antiskid } \\
\text { material }\end{array}$ & $\begin{array}{l}\text { Cobb } \\
\text { Sasso } \\
175\end{array}$ & Ross 308 & 16 birds/m2 \\
\hline $\begin{array}{l}\text { Bizeray, et al., } \\
2002 b\end{array}$ & Wooden beams & $15 \mathrm{~cm}$ & $\begin{array}{l}100 / 150 \times 4 \\
\times 15 \mathrm{~cm}\end{array}$ & - & - & Ross 308 & 10 birds $/ \mathrm{m} 2$ \\
\hline \multirow[t]{2}{*}{ Blaauw, 2019} & $\begin{array}{l}\text { Elevated } \\
\text { wooden } \\
\text { platform }\end{array}$ & $40 \mathrm{~cm}$ & $100 \times 20 \mathrm{~cm}$ & $\begin{array}{l}\text { Ramps with } \\
11.5^{\circ} \text { angles }\end{array}$ & $\begin{array}{l}\text { Hubbard } \\
\text { JA } 757\end{array}$ & Ross 308 & 10 birds/m2 \\
\hline & $\begin{array}{l}\text { Wooden } \\
\text { beams }\end{array}$ & $\begin{array}{l}4 \mathrm{~cm} \text { at } \\
\text { day } 0,8 \\
\mathrm{~cm} \text { at } \\
\text { day } 8,12 \\
\mathrm{~cm} \text { at } \\
\text { day } 14 \text {, } \\
16 \mathrm{~cm} \\
\text { from day } \\
21 \\
\text { onwards }\end{array}$ & $\begin{array}{l}100 \times 4 \times ? \\
\mathrm{~cm}\end{array}$ & Barrier-perch & & & \\
\hline $\begin{array}{l}\text { Bokkers and } \\
\text { Koene, } 2003\end{array}$ & Wooden slat & $10 \mathrm{~cm}$ & $80 \times 5 \times 5 \mathrm{~cm}$ & $\begin{array}{l}\text { Rounded upper } \\
\text { edge }\end{array}$ & JA 657* & $\begin{array}{l}\text { HI-Y } \\
\text { (Hubbard) } \\
*\end{array}$ & 4 birds/m2 \\
\hline
\end{tabular}




\begin{tabular}{|c|c|c|c|c|c|c|c|}
\hline \multirow[t]{2}{*}{ Reference } & \multirow[t]{2}{*}{ Material } & \multicolumn{3}{|c|}{ Design } & \multicolumn{2}{|c|}{ Genotype } & \multirow{2}{*}{$\begin{array}{l}\text { Stocking } \\
\text { density }\end{array}$} \\
\hline & & $\begin{array}{l}\text { Height } \\
\text { above } \\
\text { ground }\end{array}$ & $\begin{array}{l}\text { Dimensions } \\
\text { (length } \times \\
\text { width } \times \text { depth } \\
\text { OR length, } \varnothing \text { ) }\end{array}$ & $\begin{array}{l}\text { Shape and } \\
\text { access ramp }\end{array}$ & $\begin{array}{l}\text { Slow- } \\
\text { growing }\end{array}$ & $\begin{array}{l}\text { Fast- } \\
\text { growing }\end{array}$ & \\
\hline \multirow[t]{2}{*}{$\begin{array}{l}\text { De Jong and } \\
\text { Van Wijhe- } \\
\text { Kiezebrink, } \\
2014\end{array}$} & $\begin{array}{l}\text { Elevated } \\
\text { plastic (mesh) } \\
\text { platform }\end{array}$ & $\begin{array}{l}25 \mathrm{~cm} \\
\text { (low } \\
\text { platform } \\
\text { ), and } 50 \\
\mathrm{~cm} \text { (high } \\
\text { platform } \\
\text { ) }\end{array}$ & $\begin{array}{l}100 \times 100 \\
\mathrm{~cm} \text { (low } \\
\text { platform), } \\
\text { and } 500 \times \\
100 \mathrm{~cm} \\
\text { (high } \\
\text { platform) }\end{array}$ & - & $\begin{array}{l}\text { Hubbard } \\
\text { JA } 757\end{array}$ & - & 13 birds/m2 \\
\hline & Metal pipe & $\begin{array}{l}25,50 \\
\text { and } 75 \\
\mathrm{~cm}\end{array}$ & $\begin{array}{l}280 \times 110 \\
\mathrm{~cm}\end{array}$ & $\begin{array}{l}\text { A-frame with } \\
\text { perches }\end{array}$ & & & \\
\hline $\begin{array}{l}\text { De Jong and } \\
\text { Gunnink, } 2019\end{array}$ & Metal pipe & $\begin{array}{l}5 \mathrm{~cm} \text { at } \\
\text { day } 0,15 \\
\mathrm{~cm} \text { at } \\
\text { day 14, } \\
20 \mathrm{~cm} \text { at } \\
\text { day } 21 \text {, } \\
25 \mathrm{~cm} \text { at } \\
\text { day } 28 \text {, } \\
35 \mathrm{~cm} \\
\text { from day } \\
34 \\
\text { onwards }\end{array}$ & $\begin{array}{l}85 \mathrm{~m}, \\
\varnothing 5 \mathrm{~cm}\end{array}$ & Round & - & Ross 308 & 19.8 birds $/ \mathrm{m} 2$ \\
\hline $\begin{array}{l}\text { Groves and } \\
\text { Muir, } 2013\end{array}$ & Wooden beams & $\begin{array}{l}15 \text { and } \\
30 \mathrm{~cm}\end{array}$ & $\begin{array}{l}100 \times 4 \times \\
4 \mathrm{~cm}\end{array}$ & - & - & $\begin{array}{l}\mathrm{N} / \mathrm{A}, \mathrm{BW} \\
\text { at day } 28 \text { : } \\
\text { breed A } \\
1590 \mathrm{~g}, \\
\text { breed B } \\
1502 \mathrm{~g}\end{array}$ & 3.8 birds/m2 \\
\hline \multirow[t]{3}{*}{$\begin{array}{l}\text { Estevez, et al., } \\
2002\end{array}$} & $\begin{array}{l}\text { Iron pipes, } \\
\text { cooled with } 10^{\circ} \\
\mathrm{C} \text { water }\end{array}$ & $15 \mathrm{~cm}$ & $\begin{array}{l}456 \mathrm{~cm}, \\
\varnothing 3.8 \mathrm{~cm}\end{array}$ & Round & - & $\begin{array}{l}\text { Peterson } \\
\times \text { Cobb }\end{array}$ & 13.43 birds $/ \mathrm{m} 2$ \\
\hline & $\begin{array}{l}\text { Iron pipes, } \\
\text { cooled with } 10^{\circ} \\
\text { C water }\end{array}$ & $7.5 \mathrm{~cm}$ & & & & & \\
\hline & Iron pipes & $15 \mathrm{~cm}$ & & & & & \\
\hline \multirow{2}{*}{$\begin{array}{l}\text { Kaukonen, et } \\
\text { al., 2016; } \\
\text { Kaukonen, et } \\
\text { al., } 2017\end{array}$} & $\begin{array}{l}\text { Elevated } \\
\text { plastic } \\
\text { platforms }\end{array}$ & $30 \mathrm{~cm}$ & $360 \times 60 \mathrm{~cm}$ & $\begin{array}{l}\text { Ramps with } \\
15^{\circ} \text { angles }\end{array}$ & - & Ross 508 & 16 birds/m2 \\
\hline & $\begin{array}{l}\text { Wooden } \\
\text { beams }\end{array}$ & $\begin{array}{l}10 \text { and } \\
30 \mathrm{~cm}\end{array}$ & $\begin{array}{l}200 \times 5 \times \\
5 \mathrm{~cm} \text { or } 200 \\
\times 2.2 \times \\
2.2 \mathrm{~cm}\end{array}$ & $\begin{array}{l}\text { Rounded } \\
\text { upper edge }\end{array}$ & & & \\
\hline \multirow[t]{3}{*}{$\begin{array}{l}\text { LeVan, et al., } \\
2000\end{array}$} & PVC pipes & $8.5 \mathrm{~cm}$ & $\begin{array}{l}91 \mathrm{~cm} \text { (main } \\
\text { bar), } 28 \mathrm{~cm}\end{array}$ & $\begin{array}{l}\text { Without ramp, } \\
\text { round }\end{array}$ & - & $\begin{array}{l}\text { Avian } x \\
\text { Avian }\end{array}$ & 11.11 birds $/ \mathrm{m}^{2}$ \\
\hline & & $17 \mathrm{~cm}$ & $\begin{array}{l}\text { (crossbar), } \\
\varnothing 2.6 \mathrm{~cm}\end{array}$ & $\begin{array}{l}\text { Ramps with } 10^{\circ} \\
\text { angle, round }\end{array}$ & & & \\
\hline & & $35.5 \mathrm{~cm}$ & & $\begin{array}{l}\text { Ramps with } 20^{\circ} \\
\text { angles, round }\end{array}$ & & & \\
\hline $\begin{array}{l}\text { Malchow, et } \\
\text { al., } 2019\end{array}$ & $\begin{array}{l}\text { Elevated } \\
\text { plastic (mesh) } \\
\text { platforms }\end{array}$ & $\begin{array}{l}10,30, \\
50 \mathrm{~cm}\end{array}$ & $90 \times 30 \mathrm{~cm}$ & $\begin{array}{l}\text { One ramp } \\
\text { (width: } 20 \\
\mathrm{~cm}\end{array}$ & $\begin{array}{l}\text { Lohmann } \\
\text { Dual } \\
\text { (medium- }\end{array}$ & Ross 308 & 8 birds/m2 \\
\hline
\end{tabular}




\begin{tabular}{|c|c|c|c|c|c|c|c|}
\hline \multirow[t]{3}{*}{ Reference } & \multirow[t]{2}{*}{ Material } & \multicolumn{3}{|c|}{ Design } & \multicolumn{2}{|c|}{ Genotype } & \multirow{2}{*}{$\begin{array}{l}\text { Stocking } \\
\text { density }\end{array}$} \\
\hline & & \multirow[t]{2}{*}{$\begin{array}{l}\text { Height } \\
\text { above } \\
\text { ground }\end{array}$} & \multirow[t]{2}{*}{$\begin{array}{l}\text { Dimensions } \\
\text { (length } \times \\
\text { width } \times \text { depth } \\
\text { OR length, } \varnothing \text { ) }\end{array}$} & $\begin{array}{l}\text { Shape and } \\
\text { access ramp }\end{array}$ & $\begin{array}{l}\text { Slow- } \\
\text { growing }\end{array}$ & \multirow[t]{2}{*}{$\begin{array}{l}\text { Fast- } \\
\text { growing }\end{array}$} & \\
\hline & & & & $\begin{array}{l}\text { inclination } \\
\text { angle: } 35^{\circ} \text { ) } \\
\text { installed in } \\
\text { between the } \\
\text { platforms }\end{array}$ & $\begin{array}{l}\text { growing); } \\
\text { Lohmann } \\
\text { Brown } \\
\text { Classic } \\
\text { (slow- } \\
\text { growing) }\end{array}$ & & \\
\hline \multirow[t]{2}{*}{$\begin{array}{l}\text { Martrenchar, et } \\
\text { al., } 2000\end{array}$} & \multirow[t]{2}{*}{ Wooden beams } & \multirow[t]{2}{*}{$\begin{array}{l}20 \text { and } \\
33 \mathrm{~cm}\end{array}$} & \multirow[t]{2}{*}{$\begin{array}{l}200 / 400 \times \\
3.5 \times 5 \mathrm{~cm}\end{array}$} & \multirow[t]{2}{*}{$\begin{array}{l}\text { Rounded upper } \\
\text { edge }\end{array}$} & \multirow[t]{2}{*}{-} & \multirow[t]{2}{*}{ Ross $^{1}$} & $\begin{array}{l}\text { Exp. 1: } 11 \text { or } 22 \\
\text { broilers } / \mathrm{m}^{2}\end{array}$ \\
\hline & & & & & & & $\begin{array}{l}\text { Exp. } 2 \& 3: 17 \\
\text { broilers } / \mathrm{m}^{2}\end{array}$ \\
\hline \multirow[t]{2}{*}{ Nielsen, 2004} & \multirow[t]{2}{*}{ Wooden beams } & 40,65 & \multirow{2}{*}{$\begin{array}{l}75 \times 5.7 \times \\
3.8 \mathrm{~cm}\end{array}$} & \multirow[t]{2}{*}{ - } & i657 & \multirow[t]{2}{*}{-} & \multirow{2}{*}{6 birds $/ \mathrm{m}^{2}$} \\
\hline & & $\begin{array}{l}90,115 \\
\text { and } \\
140 \mathrm{~cm}\end{array}$ & & & Labresse & & \\
\hline \multirow[t]{3}{*}{$\begin{array}{l}\text { Norring, et al., } \\
2016\end{array}$} & \multirow{3}{*}{$\begin{array}{l}\text { Elevated plastic } \\
\text { platforms } \\
\text { Wooden perches }\end{array}$} & $30 \mathrm{~cm}$ & $360 \times 60 \mathrm{~cm}$ & \multirow{3}{*}{$\begin{array}{l}\text { Ramps with } 15^{\circ} \\
\text { angles } \\
\text { Rounded upper } \\
\text { edge }\end{array}$} & - & \multirow[t]{3}{*}{ Ross 508} & \multirow[t]{3}{*}{16 birds $/ \mathrm{m}^{2}$} \\
\hline & & $10 \mathrm{~cm}$ & $200 \times 5 \times$ & & & & \\
\hline & & $30 \mathrm{~cm}$ & $\begin{array}{l}5 \mathrm{~cm} \text { or } 200 \times \\
2.2 \times 2.2 \mathrm{~cm}\end{array}$ & & & & \\
\hline \multirow[t]{3}{*}{$\begin{array}{l}\text { Pettit-Riley and } \\
\text { Estevez, } 2001\end{array}$} & \multirow[t]{3}{*}{ PVC pipes } & $8.5 \mathrm{~cm}$ & \multirow{3}{*}{$\begin{array}{l}91 \mathrm{~cm} \text { (main } \\
\text { bar), } 28 \mathrm{~cm} \\
\text { (crossbar), } \\
\varnothing 2.6 \mathrm{~cm}\end{array}$} & $\begin{array}{l}\text { Without ramp, } \\
0^{\circ} \text { angle, round }\end{array}$ & \multirow[t]{3}{*}{-} & \multirow[t]{3}{*}{$\begin{array}{l}\text { Avian } x \\
\text { Avian }\end{array}$} & $\begin{array}{l}10,15 \text { and } 20 \\
\text { broilers/m² }\end{array}$ \\
\hline & & $\begin{array}{l}17 \mathrm{~cm} \\
(\max )\end{array}$ & & $\begin{array}{l}\text { Ramps with } 10^{\circ} \\
\text { angle, round }\end{array}$ & & & \\
\hline & & $\begin{array}{l}35.5 \mathrm{~cm} \\
(\max )\end{array}$ & & $\begin{array}{l}\text { Ramps with } 20^{\circ} \\
\text { angle, round }\end{array}$ & & & \\
\hline $\begin{array}{l}\text { Pettit-Riley, et } \\
\text { al., } 2002\end{array}$ & PVC pipes & $8.5 \mathrm{~cm}$ & $\begin{array}{l}91 \mathrm{~cm} \text { (main } \\
\text { bar), } 28 \mathrm{~cm}\end{array}$ & $\begin{array}{l}\text { Without ramp, } \\
0^{\circ} \text { angle, round }\end{array}$ & & $\begin{array}{l}\text { Avian } x \\
\text { Avian }\end{array}$ & $\begin{array}{l}10,15 \text { and } 20 \\
\text { broilers/m2 }\end{array}$ \\
\hline & & $\begin{array}{l}17 \mathrm{~cm} \\
(\max )\end{array}$ & $\begin{array}{l}\text { (crossbar), } \\
\varnothing 2.6 \mathrm{~cm}\end{array}$ & $\begin{array}{l}\text { Ramps with } 10^{\circ} \\
\text { angle, round }\end{array}$ & & & \\
\hline & & $\begin{array}{l}35.5 \mathrm{~cm} \\
(\max )\end{array}$ & & $\begin{array}{l}\text { Mixed: } 0^{\circ}, 10^{\circ}, \\
\text { and } 20^{\circ} \text { angle, } \\
\text { round }\end{array}$ & & & \\
\hline $\begin{array}{l}\text { Rodriguez- } \\
\text { Aurrekoetxea, } \\
\text { et al., 2014; } \\
\text { Rodriguez- } \\
\text { Aurrekoetxea, } \\
\text { et al., } 2015\end{array}$ & $\begin{array}{l}\text { Wooden } \\
\text { beams }\end{array}$ & $25 \mathrm{~cm}$ & $\begin{array}{l}50 \times 4 \times 25 \\
\mathrm{~cm}\end{array}$ & - & $\begin{array}{l}\text { Sasso } \\
\text { T44 }\end{array}$ & - & $\begin{array}{l}\text { Indoor: } 12 \\
\text { birds/m², } \\
\text { Outdoor: } 2 \\
\text { birds/m² }\end{array}$ \\
\hline $\begin{array}{l}\text { Sandilands, et } \\
\text { al., } 2009\end{array}$ & Wooden beams & N/A & $\begin{array}{l}150 \mathrm{~cm} \times ? \times \\
?\end{array}$ & $\begin{array}{l}\text { A-frame with } \\
42^{\circ} \text { angles }\end{array}$ & - & Ross 308 & $\mathrm{~N} / \mathrm{A}$ \\
\hline & & & & $\begin{array}{l}\text { A-frame with } \\
12^{\circ} \text { angles, } \\
\text { wire ramps }\end{array}$ & & & \\
\hline Su, et al., 2000 & Wooden beams & $\begin{array}{l}\text { Weeks 0- } \\
2: 10 \mathrm{~cm}\end{array}$ & $\begin{array}{l}? \times 3.5 \times \\
2.5 \mathrm{~cm}\end{array}$ & - & - & Ross 208 & 18 birds $/ \mathrm{m}^{2}$ \\
\hline & & $\begin{array}{l}\text { weeks 2- } \\
6: 25 \mathrm{~cm}\end{array}$ & $?, \varnothing 1.5 \mathrm{~cm}$ & & & & \\
\hline $\begin{array}{l}\text { Tahamtani, et } \\
\text { al., } 2018^{2}\end{array}$ & $\begin{array}{l}\text { Elevated } \\
\text { plastic }\end{array}$ & $30 \mathrm{~cm}$ & $540 \times 60 \mathrm{~cm}$ & $\begin{array}{l}\text { Ramps with } \\
14.5^{\circ} \text { angles }\end{array}$ & - & Ross 308 & 16.5 birds/m2 \\
\hline & platforms & $5 \mathrm{~cm}$ & $540 \times 60 \mathrm{~cm}$ & - & & & \\
\hline
\end{tabular}




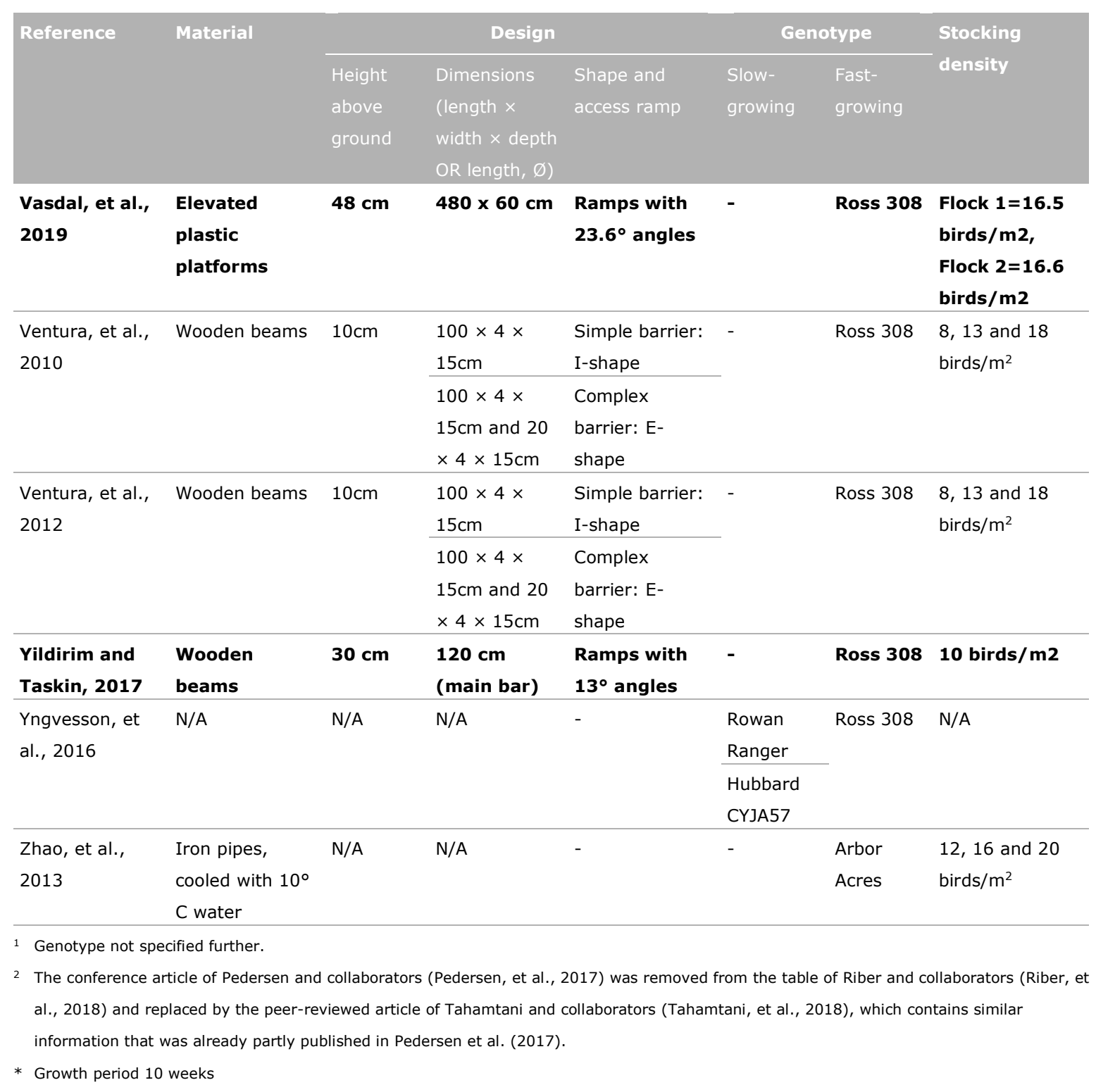

\subsubsection{Panels, Barriers, and Bales of Substrate}

Table 2 presents the main features of the panels, barriers, and bales of substrates used in the studies reviewed by Riber and collaborators (Riber, et al., 2018), updated with results from recent

publications. 
Table 2 Panel, barrier, and substrate bale design for broilers used in different studies. Adapted from Riber and collaborators (Riber, et al., 2018). Information written in bold correspond to literature published in or after the year 2017, which were added to the original table of Riber and collaborators ( $N / A=$ information not available).

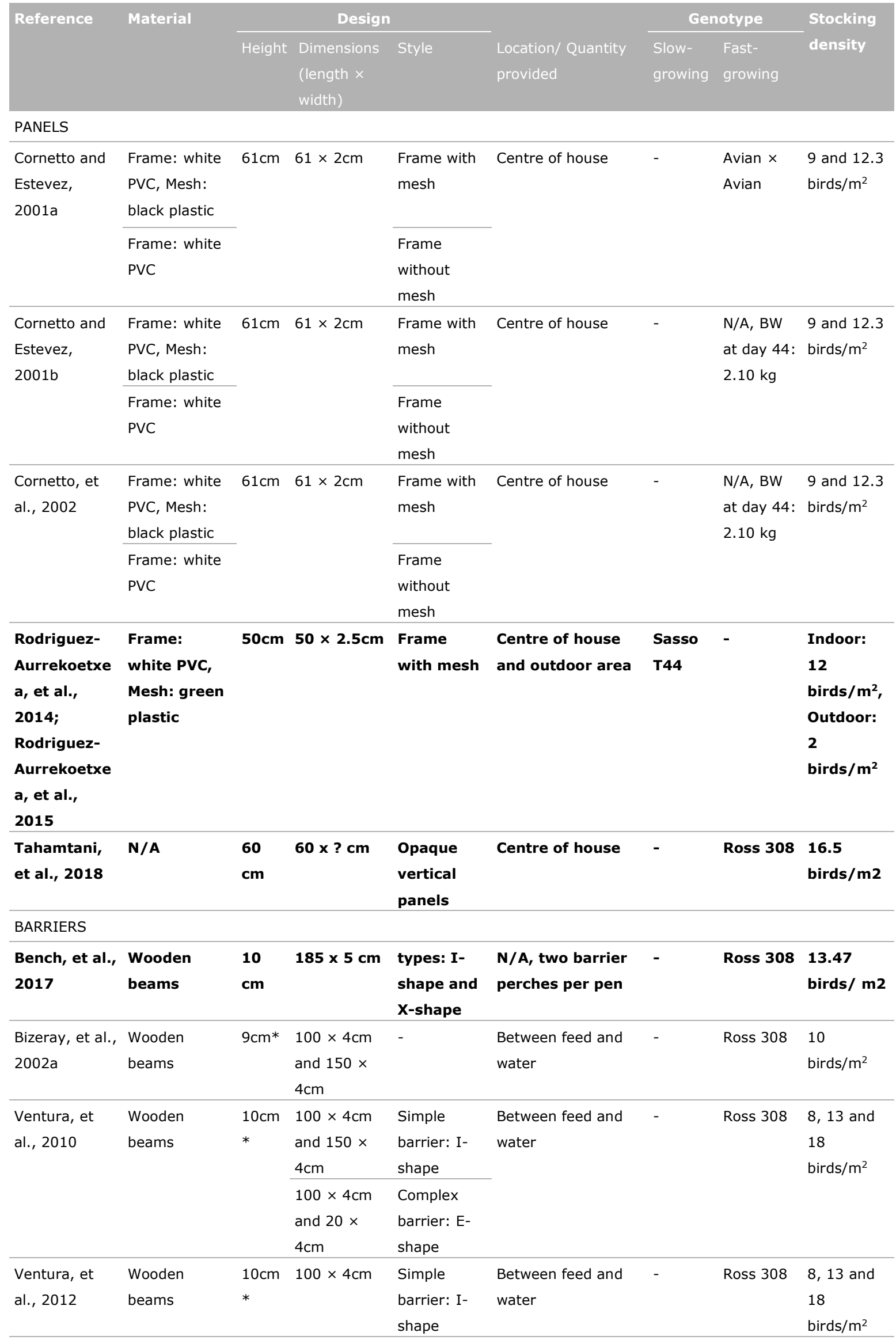




\begin{tabular}{|c|c|c|c|c|c|c|c|c|}
\hline \multirow[t]{3}{*}{ Reference } & \multirow[t]{3}{*}{ Material } & \multicolumn{3}{|c|}{ Design } & \multirow[b]{2}{*}{$\begin{array}{l}\text { Location/ Quantity } \\
\text { provided }\end{array}$} & \multicolumn{2}{|c|}{ Genotype } & \multirow{2}{*}{$\begin{array}{l}\text { Stocking } \\
\text { density }\end{array}$} \\
\hline & & Height & $\begin{array}{l}\text { Dimensions } \\
\text { (length } \times \\
\text { width) }\end{array}$ & Style & & $\begin{array}{l}\text { Slow- } \\
\text { growing }\end{array}$ & $\begin{array}{l}\text { Fast- } \\
\text { growing }\end{array}$ & \\
\hline & & & $\begin{array}{l}100 \times 4 \mathrm{~cm} \\
\text { and } 20 \times \\
4 \mathrm{~cm}\end{array}$ & $\begin{array}{l}\text { Complex } \\
\text { barrier: E- } \\
\text { shape }\end{array}$ & & & & \\
\hline \multicolumn{9}{|l|}{ SUBSTRATE } \\
\hline $\begin{array}{l}\text { Bailie, et al., } \\
2013\end{array}$ & Wheat straw & $40 \mathrm{~cm}$ & $80 \times 40 \mathrm{~cm}$ & $\begin{array}{l}\text { Bale edges } \\
\text { wrapped in } \\
\text { plastic }\end{array}$ & $\begin{array}{l}\text { Dispersed evenly, } 1 \\
\text { bale/44 } \mathrm{m}^{2}\end{array}$ & - & Ross & $\begin{array}{l}17 \\
\text { birds } / \mathrm{m}^{2} \\
\text { (but not } \\
\text { exceeding } \\
30 \mathrm{~kg} / \mathrm{m}^{2} \text { ) }\end{array}$ \\
\hline $\begin{array}{l}\text { Bailie and } \\
\text { O'Connell, } \\
2014\end{array}$ & Wheat straw & $40 \mathrm{~cm}$ & $80 \times 40 \mathrm{~cm}$ & $\begin{array}{l}\text { Bale edges } \\
\text { wrapped in } \\
\text { plastic }\end{array}$ & $\begin{array}{l}\text { Dispersed evenly, } 1 \\
\text { bale/44 } \mathrm{m}^{2} \\
\text { Dispersed evenly, } 1 \\
\text { bale/29 } \mathrm{m}^{2}\end{array}$ & - & $\begin{array}{l}\text { Ross and } \\
\text { Cobb }^{1}\end{array}$ & $\begin{array}{l}17 \\
\text { birds } / \mathrm{m}^{2} \\
\text { (but not } \\
\text { exceeding } \\
30 \mathrm{~kg} / \mathrm{m}^{2} \text { ) }\end{array}$ \\
\hline $\begin{array}{l}\text { Baxter, et } \\
\text { al., 2018b }\end{array}$ & Straw & $\begin{array}{l}40 \\
\mathrm{~cm}\end{array}$ & $80 \times 40 \mathrm{~cm}$ & $\begin{array}{l}\text { Bale edges } \\
\text { wrapped in } \\
\text { plastic }\end{array}$ & $\begin{array}{l}\text { Dispersed evenly, } \\
1 \text { bale/155 } \mathrm{m}^{2}\end{array}$ & - & Ross 308 & $\begin{array}{l}16 \\
\text { birds/m2 }\end{array}$ \\
\hline $\begin{array}{l}\text { Baxter and } \\
\text { O'Connell, } \\
2019\end{array}$ & Straw & N/A & N/A & $\begin{array}{l}\text { Bale edges } \\
\text { wrapped in } \\
\text { plastic }\end{array}$ & $\begin{array}{l}\text { Dispersed evenly, } \\
1 \text { bale/143 m2, } 3 \\
\text { bales placed in an } \\
\text { L-shape (creating } \\
\text { a semi-enclosed } \\
\text { area) }\end{array}$ & - & Ross 308 & $\begin{array}{l}16 \\
\text { birds/m2 } \\
\text { (but not } \\
\text { exceeding } \\
30 \\
\mathrm{~kg} / \mathrm{m2} \text { ) }\end{array}$ \\
\hline $\begin{array}{l}\text { Bergmann, } \\
\text { et al., } 2017\end{array}$ & Straw & N/A & N/A & $\begin{array}{l}\text { Bale edges } \\
\text { wrapped in } \\
\text { plastic }\end{array}$ & $\begin{array}{l}\text { Dispersed evenly, } \\
1 \text { bale/37 m2 }\end{array}$ & $\begin{array}{l}\text { Cobb } \\
\text { Sasso } \\
175\end{array}$ & Ross 308 & $\begin{array}{l}16 \\
\text { birds/m2 }\end{array}$ \\
\hline \multirow[t]{2}{*}{$\begin{array}{l}\text { Berghout, et } \\
\text { al., } 2018\end{array}$} & Lucerne & N/A & $\begin{array}{l}\text { N/A, } 200 \mathrm{~L} \\
\text { bales }\end{array}$ & $\begin{array}{l}\text { Plastic- } \\
\text { wrapped }\end{array}$ & $\begin{array}{l}\text { Dispersed evenly, } \\
1 \text { bale/500 birds }\end{array}$ & - & Ross 308 & $\begin{array}{l}21-23 \\
\text { birds/m2 } \\
(42\end{array}$ \\
\hline & $\begin{array}{l}\text { Wood } \\
\text { shavings }\end{array}$ & N/A & $\begin{array}{l}\text { N/A, } 800 \mathrm{~L} \\
\text { bales }\end{array}$ & $\begin{array}{l}\text { Plastic- } \\
\text { wrapped }\end{array}$ & $\begin{array}{l}\text { Dispersed evenly, } \\
1 \text { bale/500 birds }\end{array}$ & & & kg/m2) \\
\hline $\begin{array}{l}\text { De Jong and } \\
\text { Van Wijhe- } \\
\text { Kiezebrink, } \\
2014\end{array}$ & Lucerne & $\begin{array}{l}25 \text { or } \\
50 \\
\mathrm{~cm}\end{array}$ & $42 \times 55 \mathrm{~cm}$ & $\begin{array}{l}\text { Bales } \\
\text { placed in a } \\
\text { rack }\end{array}$ & $\begin{array}{l}\text { Dispersed in one } \\
\text { section (quarter) } \\
\text { of the house }{ }^{1}, 1 \\
\text { bale/ } 200 \mathrm{~m} 2\end{array}$ & $\begin{array}{l}\text { Hubbar } \\
\text { d JA } \\
757\end{array}$ & - & $\begin{array}{l}13 \\
\text { birds/m2 }\end{array}$ \\
\hline $\begin{array}{l}\text { De Jong and } \\
\text { Gunnink, } \\
2019\end{array}$ & $\begin{array}{l}\text { Wood } \\
\text { shavings }\end{array}$ & N/A & $\begin{array}{l}\mathrm{N} / \mathrm{A}, 10 \mathrm{~kg} \\
\text { bales }\end{array}$ & $\begin{array}{l}\text { Plastic- } \\
\text { wrapped } \\
\text { (but partly } \\
\text { opened) }\end{array}$ & $\begin{array}{l}\text { Dispersed evenly, } \\
1 \text { bale/34 m2 }\end{array}$ & - & Ross 308 & $\begin{array}{l}19.8 \\
\text { birds/m2 }\end{array}$ \\
\hline $\begin{array}{l}\text { Kells, et al., } \\
2001\end{array}$ & Straw & $30 \mathrm{~cm}$ & $75 \times 35 \mathrm{~cm}$ & - & 1 bale/17 $\mathrm{m}^{2}$ & - & $\begin{array}{l}\text { Ross and } \\
\text { Cobb }\end{array}$ & $\mathrm{N} / \mathrm{A}$ \\
\hline $\begin{array}{l}\text { Tahamtani, } \\
\text { et al., } 2018\end{array}$ & Straw & $\begin{array}{l}42 \\
\mathrm{~cm}\end{array}$ & $\begin{array}{l}122 \times 48 \\
\mathrm{~cm}\end{array}$ & - & $\begin{array}{l}\text { Dispersed evenly, } \\
1 \text { bale/10 } \mathrm{m} 2\end{array}$ & - & Ross 308 & $\begin{array}{l}16.5 \\
\text { birds/m2 }\end{array}$ \\
\hline $\begin{array}{l}\text { Vasdal, et } \\
\text { al., } 2019\end{array}$ & $\begin{array}{l}\text { Lucerne and } \\
\text { peat }\end{array}$ & N/A & $\begin{array}{l}\text { N/A, } 150 \mathrm{~L} \\
\text { bales of } \\
\text { peat }\end{array}$ & $\begin{array}{l}\text { Plastic- } \\
\text { wrapped } \\
\text { (but partly } \\
\text { opened) }\end{array}$ & $\begin{array}{l}\text { Dispersed evenly, } \\
1 \text { bale/67 m2 }\end{array}$ & - & Ross 308 & $\begin{array}{l}\text { Flock } \\
1=16.5 \\
\text { birds } / \mathrm{m} 2, \\
\text { Flock } \\
2=16.6 \\
\text { birds } / \mathrm{m2}\end{array}$ \\
\hline
\end{tabular}




\subsubsection{Materials and Methods Stimulating Foraging and Dustbathing Behaviour}

\section{Foraging substrates}

Table 3 presents the main features of foraging substrates used in the studies reviewed by Riber and collaborators (Riber, et al., 2018), updated with results from recent publications.

Table 3 Foraging substrates for broilers used in different studies. This table was created based on information from Riber and collaborators (Riber, et al., 2018). Information written in bold correspond to literature published in or after the year 2017, which were not present in the review of Riber and collaborators ( $N / A=$ information not available).

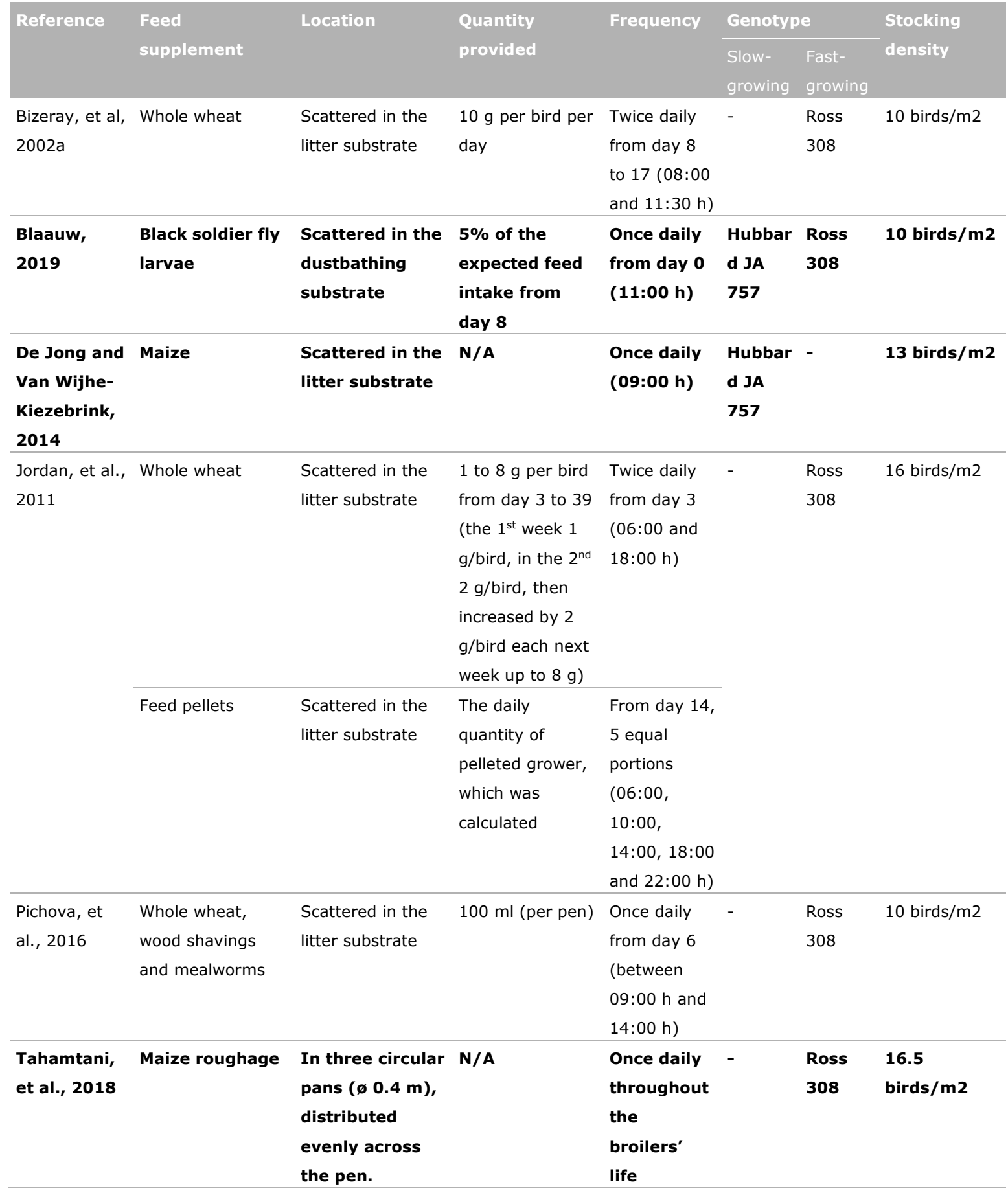




\section{Dustbathing substrates}

Table 4 presents the main features of dustbathing substrates used in the studies reviewed by Riber and collaborators (Riber, et al., 2018), updated with results from recent publications.

Table 4 Dustbathing substrates for broilers used in different studies. This table was created based on information from Riber and collaborators (Riber, et al., 2018). Information written in bold correspond to literature published in or after the year 2017, which were not present in the review of Riber and collaborators ( $N / A=$ information not available).

\begin{tabular}{|c|c|c|c|c|c|c|c|}
\hline \multirow[t]{2}{*}{ Reference } & \multirow{2}{*}{$\begin{array}{l}\text { Dustbathing } \\
\text { substrate }\end{array}$} & \multirow[t]{2}{*}{ Location } & \multirow{2}{*}{$\begin{array}{l}\text { Quantity } \\
\text { provided } \\
\text { and/or depth }\end{array}$} & \multirow{2}{*}{$\begin{array}{l}\text { Frequency } \\
\text { of refill }\end{array}$} & \multicolumn{2}{|c|}{ Genotype } & \multirow{2}{*}{$\begin{array}{l}\text { Stocking } \\
\text { density }\end{array}$} \\
\hline & & & & & $\begin{array}{l}\text { Slow- } \\
\text { growing }\end{array}$ & $\begin{array}{l}\text { Fast- } \\
\text { growing }\end{array}$ & \\
\hline $\begin{array}{l}\text { Baxter and } \\
\text { O'Connell, } \\
2016 \text {; } \\
\text { Baxter, et al., } \\
\mathbf{2 0 1 8 a}\end{array}$ & $\begin{array}{l}\text { Irish moss-peat } \\
(\mathrm{P}) \text {, oat hulls } \\
(\mathrm{OH}) \text {, straw } \\
\text { pellets (SP) or } \\
\text { wood shavings } \\
\text { (WS). }\end{array}$ & $\begin{array}{l}\text { In stainless steel } \\
\text { rings }(1.1 \mathrm{~m} \\
\text { diameter, } 7.6 \mathrm{~cm} \\
\text { deep) }\end{array}$ & N/A & $\begin{array}{l}\text { Refilled } \\
\text { throughout } \\
\text { the study } \\
\text { when more } \\
\text { than half } \\
\text { of the } \\
\text { substrate } \\
\text { was gone }\end{array}$ & - & Ross 308 & $\begin{array}{l}16 \text { birds/ } \\
\text { m2 }\end{array}$ \\
\hline $\begin{array}{l}\text { Baxter, et al., } \\
2018 b\end{array}$ & Oat hulls & $\begin{array}{l}\text { In stainless steel } \\
\text { rings ( } 1 \text { per } 155 \\
\text { m2; } 1.1 \mathrm{~m} \\
\text { diameter, } 7.6 \mathrm{~cm} \\
\text { deep), with an area } \\
\text { of } 0.95 \mathrm{~m} 2\end{array}$ & Initially 9 kg & $\begin{array}{l}\text { Refilled } \\
\text { throughout } \\
\text { the study } \\
\text { when more } \\
\text { than half } \\
\text { of the } \\
\text { substrate } \\
\text { was gone }\end{array}$ & - & Ross 308 & $\begin{array}{l}16 \text { birds/ } \\
\text { m2 }\end{array}$ \\
\hline $\begin{array}{l}\text { Baxter and } \\
\text { O'Connell, } \\
2019\end{array}$ & Oat hulls & $\begin{array}{l}\text { In stainless steel } \\
\text { rings ( } 1.1 \mathrm{~m} \\
\text { diameter, } 7.62 \mathrm{~cm} \\
\text { deep), with an area } \\
\text { of } 0.95 \mathrm{~m} 2\end{array}$ & $\begin{array}{l}\text { Initially } 14 \mathrm{~kg} \text {, } \\
\text { depth of about } \\
5 \mathrm{~cm}\end{array}$ & $\begin{array}{l}\text { Refilled } \\
\text { twice a } \\
\text { week } \\
\text { throughout } \\
\text { the study }\end{array}$ & - & Ross 308 & $\begin{array}{l}16 \text { birds/ } \\
\text { m2 (but } \\
\text { not } \\
\text { exceeding } \\
30 \mathrm{~kg} / \mathrm{m} 2 \text { ) }\end{array}$ \\
\hline $\begin{array}{l}\text { Baxter, et al., } \\
2019\end{array}$ & Moss-peat & $\begin{array}{l}\text { In steel } \\
\text { rectangles ( } 1 \text { x } 2.3 \\
\mathrm{~m}, 7.62 \mathrm{~cm} \text { deep), } \\
\text { with a total area of } \\
9.2 \mathrm{~m} 2 / \text { house }\end{array}$ & $\begin{array}{l}\text { Initially } 160 \mathrm{I} \text {, } \\
\text {, depth of } \\
\text { about } 5 \mathrm{~cm}\end{array}$ & $\begin{array}{l}\text { Refilled } \\
\text { twice a } \\
\text { week } \\
\text { throughout } \\
\text { the study }\end{array}$ & - & Ross 308 & $\begin{array}{l}16.5 \\
\text { birds/ m2 }\end{array}$ \\
\hline Blaauw, 2019 & Moss-peat & $\begin{array}{l}\text { In wooden squares } \\
(1 \mathrm{~m} \times 1 \mathrm{~m} \text { ) located } \\
\text { at the central area } \\
\text { of the pen }\end{array}$ & $\begin{array}{l}\text { Depth of } 2 \mathrm{~cm} \\
\text { in week } 1,4 \\
\text { cm in week 2, } \\
\text { and } 7.5 \mathrm{~cm} \\
\text { from week } 3 \\
\text { onwards }\end{array}$ & $\begin{array}{l}\text { Refilled as } \\
\text { needed }\end{array}$ & $\begin{array}{l}\text { Hubbard } \\
\text { JA } 757\end{array}$ & Ross 308 & $\begin{array}{l}10 \\
\text { birds/m2 }\end{array}$ \\
\hline $\begin{array}{l}\text { Vasdal, et al., } \\
2019\end{array}$ & Moss-peat & $\begin{array}{l}\text { In wooden boxes } \\
(130 \mathrm{~cm} \times 250 \mathrm{~cm} \\
\times 20 \mathrm{~cm} \text { high })\end{array}$ & $\begin{array}{l}\text { Depth of } 12 \\
\mathrm{~cm}(400 \mathrm{~L}) \text { of } \\
\text { peat }\end{array}$ & $\begin{array}{l}\text { Refilled as } \\
\text { needed }\end{array}$ & - & Ross 308 & $\begin{array}{l}\text { Flock } \\
1=16.5 \\
\text { birds } / \mathrm{m} 2, \\
\text { Flock } \\
2=16.6 \\
\text { birds } / \mathrm{m} 2\end{array}$ \\
\hline $\begin{array}{l}\text { Yildirim and } \\
\text { Taskin, } 2017\end{array}$ & Sand & $\begin{array}{l}10 \mathrm{~cm} \text { deep, } 40-\mathrm{cm} \\
\text { diameter } \\
\text { plastic black } \\
\text { container }\end{array}$ & N/A & N/A & - & Ross 308 & $\begin{array}{l}10 \\
\text { birds/m2 }\end{array}$ \\
\hline
\end{tabular}




\section{Suspended strings}

Table 5 presents the main features of suspended strings used in the studies reviewed by Riber and collaborators (Riber, et al., 2018), updated with results from recent publications.

Table 5 Suspended strings for broilers used in different studies. This table was created based on information from Riber and collaborators (Riber, et al., 2018). Information written in bold correspond to literature published in or after the year 2017, which were not present in the review of Riber and collaborators (N/A = information not available).

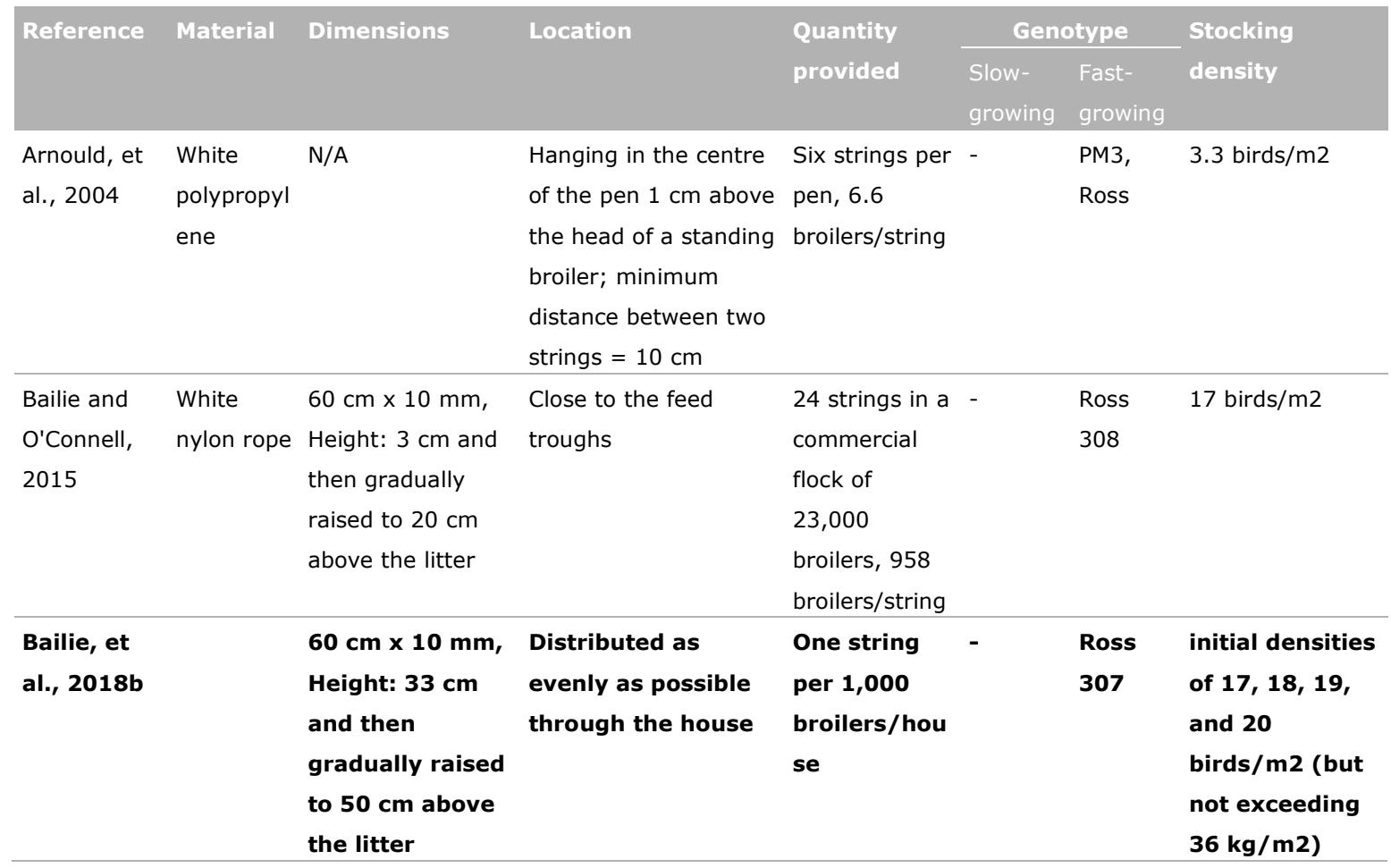

\section{Pecking objects}

Table 6 presents the main features of pecking objects used in recent studies reviewed in the current report. Thus, all information is supplementary to the review paper of Riber and collaborators (Riber, et al., 2018), which originally did not include a separate section on pecking objects.

Table 6 Pecking objects for broilers used in different studies. All information written in bold correspond to literature published in or after the year 2017, which were not present in the review of Riber and collaborators ( $N / A=$ information not available).

\begin{tabular}{|c|c|c|c|c|c|c|c|}
\hline \multirow[t]{2}{*}{ Reference } & \multirow[t]{2}{*}{ Material } & \multirow[t]{2}{*}{ Dimensions } & \multirow[t]{2}{*}{ Location } & \multirow{2}{*}{$\begin{array}{l}\text { Quantity } \\
\text { provided }\end{array}$} & \multicolumn{2}{|c|}{ Genotype } & \multirow{2}{*}{$\begin{array}{l}\text { Stocking } \\
\text { - density }\end{array}$} \\
\hline & & & & & $\begin{array}{l}\text { Slow- } \\
\text { growing }\end{array}$ & $\begin{array}{l}\text { Fast- } \\
\text { growing }\end{array}$ & \\
\hline $\begin{array}{l}\text { Baxter } \\
\text { and } \\
\text { O'Connell, } \\
2019\end{array}$ & $\begin{array}{l}\text { Black and } \\
\text { yellow } \\
\text { plastic- } \\
\text { coated } \\
\text { barrier } \\
\text { chains }\end{array}$ & $\begin{array}{l}8 \mathrm{~mm} \text {, cut to } \\
\text { lengths of } \\
\text { approximate } \\
\text { ly } 30 \mathrm{~cm}\end{array}$ & $\begin{array}{l}\text { Hung from the feeder } \\
\text { lines, in three } \\
\text { sections with two } \\
\text { hanging chains per } \\
\text { section }\end{array}$ & $\begin{array}{l}24 \\
\text { chains/house }\end{array}$ & - & $\begin{array}{l}\text { Ross } \\
308\end{array}$ & $\begin{array}{l}16 \\
\text { birds/m2 } \\
\text { (but not } \\
\text { exceeding } \\
30 \\
\mathrm{~kg} / \mathrm{m} 2 \text { ) }\end{array}$ \\
\hline $\begin{array}{l}\text { Bergmann } \\
\text {, et al., } \\
2017\end{array}$ & $\begin{array}{l}\text { Pecking } \\
\text { stones made } \\
\text { of hard- } \\
\text { pressed and }\end{array}$ & N/A & $\begin{array}{l}\text { Dispersed evenly, } 1 \\
\text { stone/60 m2 }\end{array}$ & $\begin{array}{l}34 \text { pecking } \\
\text { stones/house } \\
\text { (1.1 pecking }\end{array}$ & $\begin{array}{l}\text { Cobb } \\
\text { Sasso } \\
175\end{array}$ & $\begin{array}{l}\text { Ross } \\
308\end{array}$ & $\begin{array}{l}16 \\
\text { birds/m2 }\end{array}$ \\
\hline
\end{tabular}




\begin{tabular}{|c|c|c|c|c|c|c|c|}
\hline \multirow[t]{3}{*}{ Reference } & \multirow[t]{2}{*}{ Material } & \multirow[t]{2}{*}{ Dimensions } & \multirow[t]{2}{*}{ Location } & \multirow{2}{*}{$\begin{array}{l}\text { Quantity } \\
\text { provided }\end{array}$} & \multicolumn{2}{|c|}{ Genotype } & \multirow{2}{*}{$\begin{array}{l}\text { Stocking } \\
\text { density }\end{array}$} \\
\hline & & & & & $\begin{array}{l}\text { Slow- } \\
\text { growing }\end{array}$ & $\begin{array}{l}\text { Fast- } \\
\text { growing }\end{array}$ & \\
\hline & $\begin{array}{l}\text { dried wheat } \\
\text { bran }\end{array}$ & & & $\begin{array}{l}\text { stones per } 1000 \\
\text { birds) }\end{array}$ & & & \\
\hline \multirow{2}{*}{$\begin{array}{l}\text { Berghout, } \\
\text { et al., } \\
2018\end{array}$} & $\begin{array}{l}\text { Pecking } \\
\text { blocks }\end{array}$ & N/A & Dispersed evenly & $\begin{array}{l}1 \text { block } / 500 \\
\text { birds }\end{array}$ & - & $\begin{array}{l}\text { Ross } \\
308\end{array}$ & $\begin{array}{l}\text { 21-23 } \\
\text { birds/m2 }\end{array}$ \\
\hline & Plastic chains & $\mathbf{N} / \mathbf{A}$ & $\begin{array}{l}\text { Dispersed evenly, } \\
\text { hung from the feeder } \\
\text { lines }\end{array}$ & $\begin{array}{l}1 \text { chain } / 500 \\
\text { birds }\end{array}$ & & & $\begin{array}{l}(42 \\
\mathrm{kg} / \mathrm{m2})\end{array}$ \\
\hline $\begin{array}{l}\text { De Jong } \\
\text { and Van } \\
\text { Wijhe- } \\
\text { Kiezebrink } \\
\text {, } 2014\end{array}$ & $\begin{array}{l}\text { Pecking } \\
\text { stones }\end{array}$ & $\begin{array}{l}12 \mathrm{~cm} \\
\text { diameter }\end{array}$ & $\begin{array}{l}\text { Dispersed at equal } \\
\text { distance of about } \\
1.75 \mathrm{~m} \text { in one section } \\
\text { (quarter) of the } \\
\text { house, } 1 \\
\text { stone/160m }\end{array}$ & $\begin{array}{l}10 \text { pecking } \\
\text { stones per } \\
\text { section } \\
\text { (quarter) of the } \\
\text { house }\end{array}$ & $\begin{array}{l}\text { Hubbar } \\
\text { d JA } \\
757\end{array}$ & - & $\begin{array}{l}13 \\
\text { birds/m2 }\end{array}$ \\
\hline $\begin{array}{l}\text { De Jong } \\
\text { and } \\
\text { Gunnink, } \\
2019\end{array}$ & Metal chains & $\begin{array}{l}\text { link size } 20 \\
\times 18 \mathrm{~mm} \text {, } \\
\text { the end of } \\
\text { the chains } \\
\text { reached the } \\
\text { floor }\end{array}$ & $\begin{array}{l}\text { Attached to the two } \\
\text { ventilation channels } \\
\text { along the length of } \\
\text { the house (equally } \\
\text { distributed) }\end{array}$ & $\begin{array}{l}30 \\
\text { chains/house (1 } \\
\text { chain per } 1000 \\
\text { birds) }\end{array}$ & - & $\begin{array}{l}\text { Ross } \\
308\end{array}$ & $\begin{array}{l}19.8 \\
\text { birds/m2 }\end{array}$ \\
\hline $\begin{array}{l}\text { Yildirim } \\
\text { and } \\
\text { Taskin, }\end{array}$ & Ball & $\begin{array}{l}\text { Red plastic } \\
\text { ball (10 cm } \\
\text { diameter) }\end{array}$ & $\begin{array}{l}\text { Suspended from } \\
\text { overhead wires in } \\
\text { such a way that they }\end{array}$ & $\mathbf{N} / \mathbf{A}$ & - & $\begin{array}{l}\text { Ross } \\
308\end{array}$ & $\begin{array}{l}10 \\
\text { birds/m2 }\end{array}$ \\
\hline 2017 & Mirror & $\begin{array}{l}\text { Double faced } \\
\text { mirror }(20 x \\
10 \mathrm{~cm})\end{array}$ & $\begin{array}{l}\text { dangled from the pen } \\
\text { ceiling }\end{array}$ & $\mathbf{N} / \mathbf{A}$ & & & \\
\hline
\end{tabular}

\subsubsection{Novel objects}

There are no references available after the year 2017.

To date, there was one study conducted to assess the effects of early environmental enrichment by addition of novel objects on growth performance, fearfulness and well-being of broiler chicks (Altan, et al., 2013). Altan and collaborators (2013) added a variety of novel objects, including coloured plastic balls, plastic bottles, toys, and mirrors, from d 0 to $d 21$ to experimental pens (50 broilers/pen) housing commercial broilers (genotype and number of objects not specified). All the objects were placed on the floor, except the mirrors, which were hung from the ceiling at chicks' eye level. Every third day the objects were replaced to avoid adaptation and maintain novelty.

\subsubsection{Summing up on Point-Source Enrichments}

Table 7 summarizes the broilers' use of the different point-source enrichments reviewed and its effects on the prevalence of different welfare and production indicators measured. This table was created based on information from Riber and collaborators (Riber, et al., 2018) and literature published in or after the year 2017 taken all together in one table.

According to Riber and collaborators (Riber, et al., 2018), the opportunity to perch is utilized by broilers and will be suitable enrichment for both slow and fast-growing broilers. As such, it is an effective form of enrichment. In the original table of Riber and collaborators (Riber, et al., 2018), outcomes for animals (mostly fast-growing broilers) have been described as effects on leg/foot problems (reduced), heat stress (reduced), behaviour disturbances and aggression (reduced). The knowledge on the risk for breast blisters and keel bone damage is still low or unknown (Riber, et al., 2018). In the present table 7, some outcomes for animals have been described slightly different than in the original table of Riber and collaborators (Riber, et al., 2018). For example, the use of perches, in both fast and slow-growing broilers, was found to be low. This conclusion was based on 13 out of 20 
scientific publications reporting low use of perches in fast-growing broilers, and on 4 out of 6 scientific publications reporting low use of perches in slow-growing broilers. Particularly for the slow-growing broilers, the number of publications reporting on perch use is rather low (6 vs 20) as compared to the fast-growing broilers. In practice, slow-growing broilers seem to show much greater perch use compared to the standard fast-growing breeds (Rayner, pers. comm.). So, still more research is needed on perch use in slow-growing broilers. Also in table 7, general activity level was found to be increased in slow-growing broilers, whereas in fast-growing broilers the results were unclear, i.e. some studies show an increased activity level, and others do not. The majority of the literature reported no effects of perches on leg/foot health in both fast and slow-growing broilers. Nevertheless, in some literature perches were found to promote more even distribution of birds throughout the pen space in both fast and slow-growing broilers. Moreover, as already reported for fast-growers, perches do not seem to affect growth in the slow-growing broilers.

Although the use of perches was found to be low (particularly in fast-growing broilers), perching is considered an integral part of chicken behaviour, and is suggested to alleviate leg problems and enhance mobility in broilers, as it stimulates diversification of locomotion. Hence, there is still a need for investigation of elevated structures that are better accepted by broiler chickens (Norring, et al., 2016). As already indicated by Riber and collaborators (Riber, et al., 2018), designing perches low and wide for better support of the broilers increases usage, and perching of up to $25 \%$ of daytime has been reported in their review. Perching material and how these are provided also determines the use (e.g., metal is not preferred by birds because it is slippery). Currently, there is more information available for laying hens (EFSA, 2015) which can be used as a basis for broiler chicken studies (De Jong, pers. comm.). In addition, usage of elevated resting places is also influenced by genotype, age, environmental temperature, flock size, and stocking density (Riber, et al., 2018). These aspects should be considered in the design of future experiments.

In table 7, the use of platforms, in both fast and slow-growing broilers, was found to be frequent. General activity level was found to be increased in slow-growing broilers, whereas in fast-growing broilers the results were unclear, i.e. some studies show an increased activity level, and others do not. In slow-growing broilers, the use of platforms seems to have little influence on leg/foot health, whereas in fast growing broilers results are unclear, i.e. some studies show reduced leg/foot problems, and others do not. Some studies reporting on platform use also indicate that the slope of the ramp (that gives access to the platform) may affect leg health in broilers. Nevertheless, this aspect has not been investigated in the present report, but it merits further research in both fast and slow-growing broilers. In fast-growing broilers, a few studies have reported positive effects of platforms on decreasing fearfulness and improving welfare (Baxter, et al., 2019; Tahamtani, et al., 2018). Moreover, as already reported for fast-growers, platforms do not seem to affect growth in the slow-growing broilers.

The frequent use of platforms indicates they are better suited for broilers than perches. However, platforms did not appear to always stimulate general activity, particularly in the fast-growing broilers. The fact that the broilers used platforms to a high degree indicate that broilers are motivated to use elevated structures or driven by high animal densities. But it has been shown by De Jong and Göertz that platforms may be well used even with low stocking densities (De Jong and Göertz, 2017). It might be that the low perch use is due to physical challenges and not to a lack of motivation to use elevated structures (Norring, et al., 2016).

According to Riber and collaborators (Riber, et al., 2018), platforms offer additional possibilities for locomotion. It is possible that broilers make better use of these types of elevated resting places as compared to perches, as raised platforms will be easier to access and heavy birds may have fewer problems with finding their balance when resting. However, there is still very limited scientific knowledge, particularly in slow-growing broilers, about the effects of such resting places on behaviour and other welfare aspects. As for the perches, there is still research needed on the optimum height, dimensions and material used in platforms and ramps for slow-growing broilers.

Panels, barriers, and bales (either straw, lucerne hay or wood-shavings) are all types of environmental enrichment that broilers use for perching or for a quiet resting area to lie against with 
reduced disturbance, and as such, can be effective enrichment (Riber, et al., 2018). Particularly bales are well used by both fast and slow-growing broilers (table 7). It should be highlighted that bales are used in terms of both perching and clustering around the bales, therefore they provide additional benefits above just providing an elevated resting space (De Jong and Gunnink, 2019). To date, no studies have specifically documented the multiple uses of one enrichment, which could be considered as providing for multiple behavioural needs of broilers. So, this merits further research in both fast and slow-growing broilers. Furthermore, panels and barriers can contribute to a more even distribution of birds in the house, which ameliorates potential localized problems associated with high stocking densities. Nevertheless, scientific knowledge is unclear about effects of these objects on leg/foot health and on activity (Riber, et al., 2018). Recently, a few studies have reported positive effects of combining bales of straw or lucerne hay with other enrichment on reducing leg/foot problems in fastgrowing broilers (Baxter, et al., 2018b; Vasdal, et al., 2019), and in slow-growing broilers, bales of straw have been found to increase activity levels (Bergmann, et al., 2017), whereas in fast-growing broilers some studies show an increased activity level, and others do not.

According to Riber and collaborators (Riber, et al., 2018), the provision of a foraging substrate such as sand that is preferred by broilers will stimulate its usage and can lead to increased foraging, thereby reducing inactivity. Offering different dustbathing substrates in smaller local quantities can stimulate dustbathing and foraging in preferred substrates (moss-peat and oat hulls) (Baxter, et al., 2018a). Recently, an oat hulls substrate has been considered a successful enrichment in terms of improving bird leg health (Baxter, et al., 2018b) and a moss-peat substrate has been considered successful in reducing fearfulness in fast-growing broilers (Baxter, et al., 2019). More research is needed on the use of dustbathing substrates as effective enrichment in slow-growing broilers.

Scattering all of the broilers feed in the bedding, rather than offering it in feeders stimulates activity of fast-growing broilers, but as a consequence reduces body weight (Jordan, et al., 2011). Scattering of additional food items, such as whole wheat, in the bedding, has not been found to be effective, as it did not influence time budgets, mortality, or other welfare parameters (Bizeray, et al., 2002a).

Recently, the provision of live Black soldier fly larvae in the litter once daily has yielded positive effects on activity levels of both fast and slow-growing broilers (Blaauw, 2019). Therefore, the use of food based enrichment, such as larvae that are considered as a highly valued food by broilers, may be an effective form of enrichment, which deserves further research ${ }^{1}$.

The limited knowledge on providing suspended strings as foraging or pecking objects, shows that their use varied and their effect (if any) was limited (Riber, et al., 2018). Recently, other pecking objects such as plastic chains (Baxter and O'Connell, 2019), metal chains (De Jong and Gunnink, 2019) and pecking stones made of hard-pressed wheat bran (Bergmann, et al., 2017) were found to be well used by fast-growing broilers. However, scientific knowledge is unclear about effects of these objects on leg/foot health and on activity. In slow-growing broilers, pecking stones combined with other enrichment have been found to increase activity levels (Bergmann, et al., 2017), whereas in fast-growing broilers some studies show an increased activity level, and others do not. Moreover, an important aspect of non-food based enrichment aimed at stimulating foraging and pecking behaviour is that it should remain interesting to the birds throughout the entire rearing phase. This aspect should be considered in the design of future experiments for both fast and slow-growing broilers.

\footnotetext{
${ }^{1}$ The Dutch SPA stresses the fact that there is insufficient knowledge on insect welfare when these are reared to be used as feed for chickens, and they therefore do currently not support this type of enrichment for broiler chickens.
} 
Table 7 A summary of broilers' use of the different kinds of point-source environmental enrichment reviewed in the present review and its effect on the prevalence of different welfare and production indicators measured. "Fast" and "slow" refer to growth rate. Adapted from Riber and collaborators (Riber, et al., 2018).

\begin{tabular}{|c|c|c|c|c|c|c|c|c|c|c|c|}
\hline \multirow[t]{2}{*}{ Type } & \multirow[t]{2}{*}{ Genotype } & \multirow{2}{*}{$\begin{array}{l}\text { Use by the } \\
\text { birds }\end{array}$} & \multicolumn{9}{|c|}{ Effect on } \\
\hline & & & Activity level & $\begin{array}{l}\text { Leg/foot } \\
\text { problems }\end{array}$ & Heat stress & Fear level & $\begin{array}{l}\text { Distribution in } \\
\text { house }\end{array}$ & Disturbances & Growth & Breast blisters & $\begin{array}{l}\text { Keel bone } \\
\text { damages }\end{array}$ \\
\hline \multirow[t]{2}{*}{ Perches } & Fast & Little & Unclear $^{1}$ & No effect & Reduced & No effect & Improved & Reduced & No effect & - & Increased \\
\hline & Slow & Little & Increased & No effect & - & - & Improved & - & No effect & Increased & Increased \\
\hline \multirow[t]{2}{*}{ Platforms } & Fast & Well used & Unclear $^{1}$ & Unclear $^{2}$ & - & Reduced & - & - & Unclear $^{3}$ & - & - \\
\hline & Slow & Well used & Increased & No effect & - & - & - & - & No effect & - & - \\
\hline \multirow[t]{2}{*}{ Panels } & Fast & Well used & Reduced & - & - & - & Improved & Reduced & No effect & - & - \\
\hline & Slow & - & - & - & - & - & Improved & - & - & - & - \\
\hline Barriers & Fast & Moderate & No effect ${ }^{4}$ & Reduced & - & Unclear $^{5}$ & - & Reduced & No effect & - & - \\
\hline \multirow{2}{*}{$\begin{array}{l}\text { Bales of straw, Lucerne or } \\
\text { wood-shavings }\end{array}$} & Fast & Well used & Unclear $^{1}$ & Reduced & - & Unclear ${ }^{6}$ & - & No effect & No effect & - & - \\
\hline & Slow & Well used & Increased & - & - & - & - & - & - & - & - \\
\hline \multirow{2}{*}{$\begin{array}{l}\text { Foraging substrates } \\
\text { (supplement) }\end{array}$} & Fast & Well used & Unclear $^{1}$ & - & - & - & - & - & - & - & - \\
\hline & Slow & - & Increased & No effect & - & - & - & - & No effect & - & - \\
\hline $\begin{array}{l}\text { Whole wheat (supplement) } \\
\text { spread in bedding }\end{array}$ & Fast & - & No effect & Increased $^{7}$ & - & No effect & - & - & No effect & - & - \\
\hline All feed spread in bedding & Fast & - & Increased & - & - & - & - & - & Reduced & - & - \\
\hline \multirow[t]{2}{*}{ Dustbathing substrates } & Fast & Well used & Increased & Reduced & - & Reduced & - & No effect & Unclear $^{3}$ & - & - \\
\hline & Slow & Little & Increased & No effect & - & - & - & - & No effect & - & - \\
\hline Suspended strings & Fast & Little & Unclear $^{1}$ & Reduced & - & No effect & - & - & No effect & - & - \\
\hline \multirow[t]{2}{*}{ Pecking objects } & Fast & Well used & Unclear $^{1}$ & Unclear $^{2}$ & - & No effect & - & No effect & No effect & - & - \\
\hline & Slow & Unclear $^{8}$ & Increased & - & - & - & - & - & - & - & - \\
\hline Novel objects & Fast & - & - & - & - & Reduced & - & - & - & - & - \\
\hline
\end{tabular}

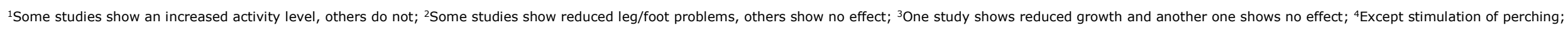
${ }^{5}$ Simple barriers increased susceptibility to the induction of $\mathrm{TI}$ compared to complex barriers or no barriers; ${ }^{6} \mathrm{One}$ study shows reduced fear level and another one shows no effect; ${ }^{7} \mathrm{One}$ study shows higher gait scores in comparison to a control group; ${ }^{8}$ One study shows that pecking objects are well used and another one shows little use. 


\subsubsection{More Complex Enriched Environments with Outdoor Access}

There are no references available after the year 2017.

As already reviewed by Riber and collaborators (Riber, et al., 2018), studies on whether an outdoor area (or free range) offers an enriched environment for broilers are limited, but show that range use is stimulated by good cover on the range (preferably mature trees with good canopy cover, combined with a mixture of grass, lower vegetation, and nutritious plants) and beneficial weather conditions. Furthermore, range use increases with increasing bird age. Slow-growing breeds appear to benefit most from an outdoor area, as they show higher levels of use, activity, and travel further outside than fast-growing breeds. This benefit also manifests itself in better foot pad health and plumage condition compared to slow-growing birds that are kept indoors. Information on mortality and parasite risks for broilers is very limited.

\subsubsection{More Complex Enriched Environments: The Higher Welfare Indoor Systems}

There are no references available after the year 2017.

As already reviewed by Riber and collaborators (Riber, et al., 2018), the scientific knowledge on the effects of higher welfare indoor systems on broiler health and welfare is very limited, although these systems are increasingly used in practice. Briefly, these are relatively new systems with animal welfare standards positioned between conventional and organic broiler production systems (Riber, et al., 2018). Originally, the aim of these systems was to improve animal welfare with relatively moderate technical changes and, consequently, a moderate increase in production costs (Saatkamp, et al., 2019). They often use broiler breeds with a lower growth potential as compared to conventional systems or they use fast-growing breeds but have a lower maximum stocking density. Frequently, the environment in these production systems is richer in stimuli as compared to conventional systems, e.g., perches, substrate bales, pecking objects, and natural light (windows) are provided. They may also have a covered veranda, which provides a halfway house between the indoor and outdoor environment and provides scratching opportunities. Prevalence of welfare problems such as contact dermatitis, lameness, and heat stress is considered to be lower in these systems as compared to conventional systems (EFSA, 2010).

\subsection{Practical inventory}

\subsubsection{Overview of the requirements for the use of enrichment in the different broiler production systems in the Netherlands and United Kingdom}

Several United Kingdom and Dutch retailers now produce their own-label broilers in higher welfare indoor systems. For an overview of the Dutch production systems, refer for instance to Ellen and collaborators (Ellen, et al., 2012). Such systems offer enrichment, some in combination with natural light (windows) and often with a maximum stocking density in place. Some of these retailers (e.g., M\&S in the UK) will still use a fast-growing breed in these higher welfare indoor systems, whereas all retailers in The Netherlands already use a slower-growing breed (Saatkamp, et al., 2019). Several large retailers, such as Albert Heijn and Jumbo that are market leaders in the Netherlands, are no longer supplying conventional fresh broiler meat and have replaced it with a new product from their own concept with (claimed) higher animal welfare (Saatkamp, et al., 2019). Differences exist between these new retail standard concepts developed in the United Kingdom and in the Netherlands, but common characteristics include:

- a more robust, slower growing breed, such as Hubbard JA757;

- a maximum average daily growth of 50 grams;

- $\quad$ indoor housing with more space, and at relatively lower stocking densities ( $\leq 38 \mathrm{~kg} / \mathrm{m} 2)$;

- $\quad$ provision of enrichment, and an adapted light regime (sometimes including natural light).

In Table 1 (The Netherlands) and Table 2 (United Kingdom) of Appendix 1 the different retailer concepts are explained in more quantitative detail. For the purpose of the current report, the description of the different concepts was limited to the requirements for the provision of daylight, dark 
period, use of enrichment, breeds and stocking density. In most systems natural daylight should be provided in at least 3\% of the useable area in the broiler house, the dark period should be of at least 6 continuous hours ( 8 hours is required in a few systems), and the requirements for the use of enrichment often include substrate bales, pecking objects, scattered grains, platforms or perches, but in most systems substrate bales are required. Specific information on breeds and stocking density in given in Appendix 1.

\subsubsection{List of enrichments that are commonly applied into practice in the different broiler production systems in the Netherlands}

Based on consultation of stakeholders, enrichments that are currently used in slow-growing broiler production systems in the Netherlands include: substrate bales, pecking stones, scattering of additional food items (such as whole wheat in the bedding), perches and platforms. In practice, there is not much variation in the use of enrichments. It seems that regularly one or two types of enrichment are used per farm, and mostly indoors. Substrate bales and additional food items in the bedding are types of enrichments that are often combined.

Regarding substrate bales, compact bales of Lucerne $(20 \mathrm{~kg}$, pressed, tied with 4 straps and sometimes provided inside a nylon feeding net) are currently used at circa $20-25 \%$ of the farms that provide enrichment in the form of substrate bales. Some reasons for its limited use may include costs, because a bale of Lucerne is usually more expensive and is more quickly consumed by the chickens than a bale of straw. Old birds are able to consume large amounts of Lucerne, which may interfere with the intake of their regular diet. Moreover, Lucerne is retained in the gizzard for a long period of time, which may interfere with the period of fasting normally applied prior to slaughter. Therefore, in the majority of the farms (circa 75\%) bales of straw are currently applied. Generally, long straw is chopped $(4-5 \mathrm{~cm})$, very tightly pressed and tied in small bales. The quantity provided corresponds to 1 bale per 1000 birds, but some (supermarket) concepts allow 1 bale per 2000 birds. The amount of kilos per bale depends also on the concept, but $20 \mathrm{~kg}$ is usually the minimum. The bales stay from the start until the end of the rearing period in the broiler house, and may be replaced during this period if, for instance, the top of a bale collapses after birds have been pecking on it. If a bale does not collapses by itself prior to slaughter, it is often broken down by the farmer so that it can spread in the bedding in the week before the slaughter. Alternatively to straw, chopped rapeseed straw or wood shavings (large curls) are also used as substrate for bales. Future research is needed to look at the availability, cost and sustainability of variable substrates.

Regarding pecking stones, mineral pecking stones of different hardness grades are commonly used for laying hens and are now being used for broiler chickens as well. The quantity provided varies according to the requirements from the different (supermarket) concepts.

Scattering of additional food items (such as whole wheat, at least 2 grams per bird per day) is also used in some broiler farms to increase foraging activity of the chicken. In practice, it does not seem to increase foraging activity of broiler chickens, and, if needed, it may be used to train the chickens to go inside the broiler house.

Regarding perches and platforms (without ramps), although required by some (supermarket) concepts (see Tables 1 and 2 in Appendix 1), their use by broiler farmers is also considered limited at the moment. When applied, particularly perches are not well used by regular fast-growing broiler chickens. Only the chickens that are lagging behind in growth seem to use the perches more frequently than the chicken with normal growth. In practice, bales of straw seem to be more frequently used as elevated resting places than perches by the broiler chickens. Moreover, chickens are often seen clustering around the bales. Thus, bales may have other functions than only perching.

Interestingly, hanging ribbons (long, fluttering in the air stream) throughout the broiler house before the chicks enter the house for the first time seem to reduce fearfulness. Some farmers use ribbons to habituate the chickens to the occurrence of movements coming from above in the broiler house, which may reduce the occurrence of fearfulness reactions. A radio is also sometimes used in the broiler house to avoid sudden panic reactions of the chickens. 
The majority of the farmers use moss-peat as litter (60-70\%). Compressed straw pellets, wood shavings and chopped straw are also used. 


\section{$4 \quad$ Results on Natural Light}

\subsection{Literature study}

Commercial broiler production systems based on retailers initiatives to improve animal welfare above the minimum legal requirements have emerged in several European countries, such as the United Kingdom and the Netherlands (Saatkamp, et al., 2019; Vissers, et al., 2019). A common factor in the higher welfare indoor systems is the application of environmental enrichment, with or without natural light, or systems with a covered veranda or outdoor range, to stimulate locomotor activity and natural behaviours of the broiler chickens (De Jong and Gunnink, 2019). Requirements for the provision of natural day light (at least $3 \%$ of the useable area) and use of enrichment in higher welfare indoor systems used for broiler production in The Netherlands are given in Appendix 1.

Recently, it has been reported that in commercial houses with fast-growing broilers, provision of environmental enrichment (wood shavings bales, perches and metal chains) and natural light promoted bird activity more than providing environmental enrichment without natural light, or providing no enrichment (De Jong and Gunnink, 2019). Moreover, the combination of natural light and straw bales not only increased locomotor activity but also improved leg health in fast-growing broilers, as compared with flocks without natural light and straw bales and flocks with straw bales but without natural light (Bailie, et al., 2013). When perches and strings were combined with straw bales in houses with natural light, there was a negative effect on fast-growing broiler activity in areas away from the enrichments (Bailie and O'Connell, 2015), which may suggest that there were functional areas separating resting and active chickens. In another study, where the effect of an outdoor run and natural light on the welfare of fast-growing broilers was investigated, it was concluded that an outdoor run improved welfare of broilers more than natural light alone, but still more research is needed on the quality and intensity of lighting provided to broiler chickens (Ruis, et al., 2004). With regard to intensity, it has been suggested that specifically, the variation in light intensity is an important driver of the stimulating effects of natural light on broiler activity (Bailie, et al., 2013). This not only stimulates activity but is also suggested to better synchronise flock behaviour, which may lead to higher activity in the photoperiod and more uninterrupted resting in the dark period (Alvino, et al., 2009). Also, variation in light intensity across the broiler house may stimulate birds to use different areas around the house for different behaviours during the photoperiod, but this depends largely on the method of providing natural light. This means that the way in which windows are incorporated into broiler houses is also important, but this has not been investigated yet. All in all, there is still limited scientific knowledge about the effects of natural light (alone or in combination with enrichments) provision on behaviour and welfare of broilers, both of fast and slow-growing breeds. Though also relevant, scientific literature on health benefits of natural light (e.g., via endogenous synthesis of vitamin $\mathrm{D}$ ) is not included in the current report, because it falls outside the scope of the study. 
Table $8 \quad$ Natural light for broilers used in different studies.

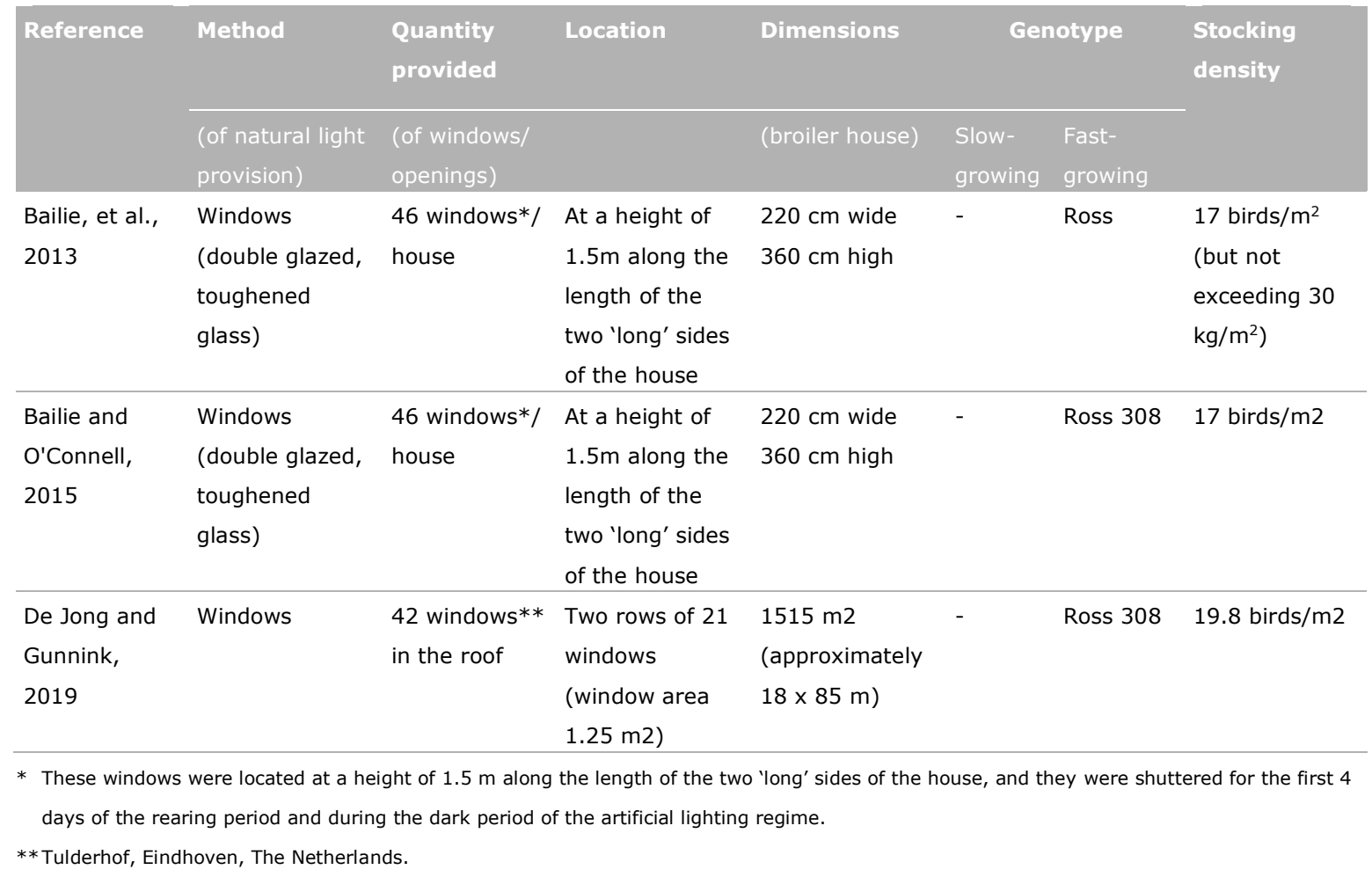

\subsection{Practical inventory}

Based on consultation of stakeholders, provision of natural light is variable among the different slowgrowing broiler production systems in the Netherlands. In organic broiler production systems, chickens are provided with an outdoor access (free range) or in the so-called 'Beter Leven' 1 star system, broiler houses are provided with a covered outdoor area ("veranda") which gives direct access to natural light. In indoor systems, most broiler houses are equipped with windows in the roof slope enabling entrance of natural light in the house. Nevertheless, natural light can be provided in different ways. For example, through glass windows in the walls, polycarbonate black out roof windows, polycarbonate black out wall or roof ridges which can be (partially) closed using slats or screens. With slats, it is usually difficult to completely ban the light entry. If windows are in the walls there is usually less direct sunlight in the house and light entry will be more diffuse. In this way, it is also possible to provide a lot of variation in light intensity across the broiler house.

Normally, there should be a scheme for introducing natural light in the broiler house, because young chicks tend to avoid direct day light, and may smother on the areas of shadow along the sides of the broiler house walls. Therefore, in the first week of the rearing period windows are kept closed, and artificial lights are used (10 lux or higher). An alternative could be the provision of dark brooders, such as in the 'Windstreek' system. After one week, the farmer starts with natural light provision (25-50\% of capacity). If there is a lot of sun (high light intensity), the amount of natural light provided is kept low, and is gradually increased. If it is very sunny, farmers use the screens or slats to dim the light. When sensors are used, there is quite some variation according to the outside light conditions. Artificial lights are turned off during daytime, but may be temporarily turned on or used in addition to windows, if for instance weather conditions (clouds, rain, snow) hinder the entrance of day light in the house. With regard to the dark period, in practice, a period of 6 hours (retail concepts) or 8 hours ('Beter leven) of continuous darkness is required, but there is still a question on whether this period should be shorter or divided into more periods, to assure sufficient gut fill until the next light period. 


\section{$5 \quad$ Results on Artificial Light}

\subsection{Literature study}

\subsubsection{Effects of light during incubation}

Commercial broiler chicken eggs are often incubated in complete darkness, receiving light only intermittently when the incubator is opened, both to save electricity and because of concerns about potential negative effects on hatchability due to heat coming from the light source. Under natural conditions, however, chicken embryos would receive at least some light stimulation during development when the hen leaves the nest to feed or responds to distress calls from other chickens by rising to turn the eggs (Archer and Mench, 2014).

According to a series of peer-reviewed papers published by Archer and collaborators (e.g. Archer, 2017; Archer and Mench, 2013; Archer and Mench, 2014; Archer, et al., 2009), light stimulation during incubation can affect the behaviour and health of broiler chickens post-hatch. For example, light exposure during incubation has been shown to reduce fear and stress responsiveness post-hatch in broiler chickens, which can be beneficial for welfare (Archer and Mench, 2013; Archer and Mench, 2014). Moreover, providing light during incubation has no negative effect on production or health of broilers (Archer, et al., 2009). Recently, it has been shown that exposing broiler eggs to white light and red light that is a component of it are possibly the key spectrum to improving hatchability and lower fear and stress susceptibility, whereas green light is not as effective during incubation (Archer, 2017). These studies have been conducted with eggs from fast-growing broilers, and it would be interesting to investigate how light provision during incubation of eggs from slow-growing broilers may affect behaviour and welfare of these birds in the post-hatch rearing period.

\subsubsection{Effects of ultraviolet wavelengths on chicken behaviour}

There is increasing evidence that birds use ultraviolet (UV) signals in visually mediated behaviours, in both intraspecific signalling and foraging decisions (Maddocks, et al., 2001). In chickens, UVA wavelengths (as low as $360 \mathrm{~nm}$ ) are visible and may facilitate interactions within the flock. UVB wavelengths (290-320 nm), although not visible to chickens, promote endogenous vitamin D synthesis, which could support the rapid skeletal development of fast-growing broiler chickens. Despite this, standard housing of broiler chickens is usually indoors and without exposure to UV or natural light throughout the whole rearing period. Although windows may be incorporated into broiler houses, glass does not typically transmit any UVB wavelengths of light and the transmission of UVA wavelengths is limited depending on the type of glass used. Consequently, light from windows may not be representative of sunlight and/or does not appear "natural" to a chicken (James, et al., 2018). Given the importance of UVA as a component of chicken visual feedback, the provision of these wavelengths could be considered an effective form of environmental enrichment. To date, not many studies have assessed the impacts of artificial lighting regimes including UV wavelengths on the behaviour and welfare of broiler chickens.

In laying hens, Ruis and collaborators (Ruis, et al., 2010) found several positive outcomes with UVA, such as increased preening and ground pecking, reduced fearfulness and reduced gentle feather pecking. Similarly, Kristensen and collaborators (Kristensen, et al., 2007) showed that six-week old broiler chickens performed more preening, object manipulation, foraging, and walking when reared in lighting conditions that included some UVA. Maddocks and collaborators (Maddocks, et al., 2001) found significantly lower baseline levels of corticosterone together with a non-significant trend for increased exploratory behaviours in layer chickens (Lohmann breed) that were provided with UVA. However, not all outcomes were positive: when laying hens were reared to 50 weeks of age, Ruis and collaborators (Ruis, et al., 2010) found that UVA increased incidence of severe feather pecking at certain ages. This was reduced in all lighting treatments after the introduction of substrate. Therefore, 
Ruis and collaborators (Ruis, et al., 2010) proposed that UVA may have made the feathers of conspecifics look more appealing than in standard lighting, attracting more severe pecking in an environment lacking other stimuli.

UVA provision alone may not be a quick solution for welfare problems such as feather pecking, but it may improve the quality and the reliability of visual feedback as perceived by the chicken, potentially enhancing the appearance of both conspecifics and their environment. Particularly in coloured birds, it may increase visibility of plumage patterns. The studies mentioned above suggest that unless animals are housed in otherwise barren environments, UVA wavelengths can potentially facilitate more harmonious interactions within the flock and promote the expression of natural behaviours. Floorhoused broiler chickens are therefore a good candidate for investigating the impacts of UV wavelengths on behaviour and welfare, as they are typically provided with substrate (litter) and have a short rearing period before the onset of maturity, which means that feather pecking is not an issue (James, et al., 2018). To date, there was only one study assessing the effects of UV wavelengths on welfare indicators of fast-growing broilers (see Table 9), and there are no studies that have assessed the effects of UV wavelengths in combination with environmental enrichments on behaviour and welfare of fast and slow-growing broilers. Thus, this merits further research.

Table 9 Effects of UV wavelengths on different welfare indicators measured in fast-growing broilers reared under commercially representative conditions (N/A = information not available).

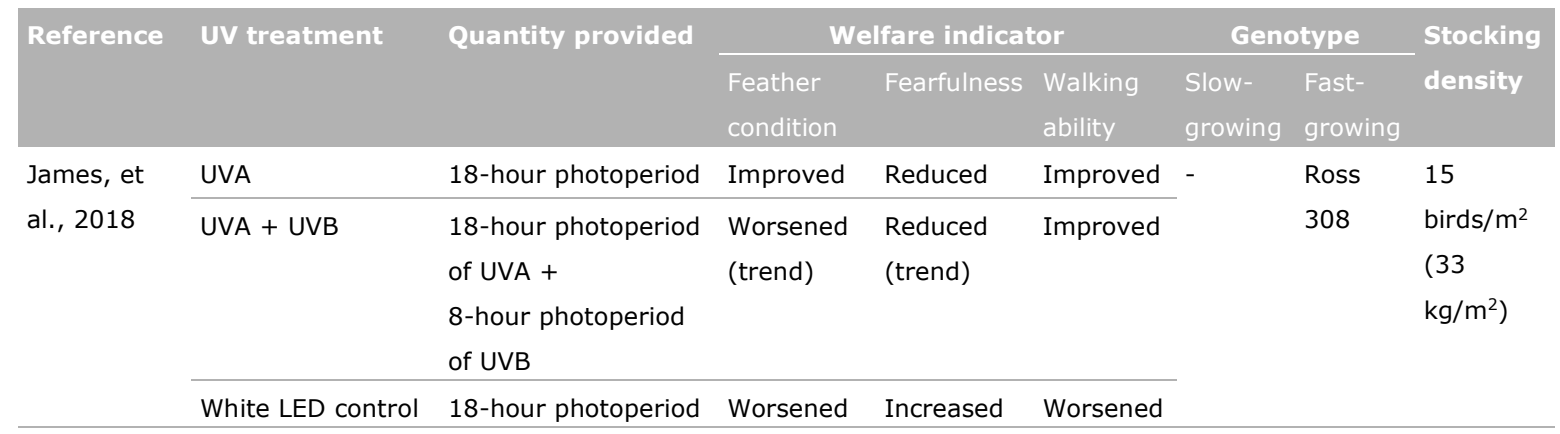

\subsubsection{Light colour preferences of broiler chickens}

Light colour is determined by different wavelengths in the spectrum. In broiler production white light (consisting of a combination of all the colours of the visible light spectrum) is often used. However, the composition of different wavelengths in white light can differ, resulting in apparent white light but with a different colour temperature. For instance, a 'warm' white light has a lower colour temperature (Kelvin) than 'cold' white light. The spectrum visible to chickens differs from humans. The eye of the chicken is more sensible to red and blue light (Niekerk, et al., 2015).

Usually, white fluorescent light is used in broiler chicken houses, but with the recent developments of LED lighting and consequently the switch from light bulbs towards LED in poultry housing, there has been a shift of attention to effects of the light spectrum (colour of the light) on broiler performance and welfare (e.g., Archer, 2018; Huth and Archer, 2015; Riber, 2015). With LED lighting, broiler chickens can be provided with a more complex and different light spectrum during the rearing period (Archer, 2018), which makes it possible to precisely adjust the light spectrum to the needs of the birds and potentially improve their welfare and performance. However, relatively little is known on the preference of broiler chickens for certain light colours or colour temperatures. It is therefore important to collect more information on the preference and behavioural responses of broiler chickens under varying light conditions, to develop light programs that promote broiler welfare.

There are indications that broiler chickens from three weeks of age onwards prefer cold white light $(6065 \mathrm{~K})$ rather than warmer white light $(4100 \mathrm{~K})$, measured by the time broiler chickens spent in the compartment with cold white light on days 16, 28 and 34 of the rearing period (Riber, 2015). 
Moreover, resting behaviour occurred more often in cold white light than in warmer white light (Riber, 2015). In another study, Mendes and collaborators (Mendes, et al., 2013) found that broiler chickens from three weeks of age onwards had a higher feed intake in white compared to yellow LED light. Other research indicated that broilers housed under cold white light $(5000 \mathrm{~K})$ for the whole rearing period were less anxious and grew faster than broiler chickens housed under warm white light (2700 K) (Archer, 2018). Studies in humans show that preference for light temperature differ depending on the activity and time of the day (Philips, pers. comm.). However, this was only included to a very limited extent in the experiment of Riber (2015) and not included in the study of Archer (2018). Thus, it is not known yet whether or not light colour preference depends on the behaviour the chicken intends to perform. In addition, the preference of broiler chickens was only included in very few studies comparing two light colour temperatures (Mendes, et al., 2013; Riber, 2015).

Table 10 Significant effects of colour lighting treatments in broiler chickens $(N / A=$ information not available).

\begin{tabular}{|c|c|c|c|c|c|c|c|c|}
\hline \multirow[t]{2}{*}{ Reference } & \multirow[t]{2}{*}{ Treatment } & \multirow{2}{*}{$\begin{array}{l}\text { Preference } \\
\text { (if tested) }\end{array}$} & \multicolumn{3}{|c|}{ Effect on } & \multicolumn{2}{|c|}{ Genotype } & \multirow{2}{*}{$\begin{array}{l}\text { Stocking } \\
\text { density }\end{array}$} \\
\hline & & & $\begin{array}{l}\text { Behaviour and } \\
\text { welfare }\end{array}$ & Growth & $\begin{array}{l}\text { Locomotion } \\
\text { disorders }\end{array}$ & $\begin{array}{l}\text { Slow- } \\
\text { growing }\end{array}$ & $\begin{array}{l}\text { Fast- } \\
\text { growing }\end{array}$ & \\
\hline $\begin{array}{l}\text { Prayitno, et } \\
\text { al., 1997b }\end{array}$ & $\begin{array}{l}\text { Red or blue } \\
\text { light at three } \\
\text { intensities } \\
\text { from } 1 \text { to } 35 \\
\text { days of age } \\
\text { (exp. } 1 \text { ) }\end{array}$ & - & $\begin{array}{l}\text { Activity } \\
\text { increased with } \\
\text { intensity in } \\
\text { red but not } \\
\text { blue light }\end{array}$ & - & - & - & Ross & $\begin{array}{l}7.5 \\
\text { birds/m2 }\end{array}$ \\
\hline $\begin{array}{l}\text { Prayitno, } \\
\text { Phillips and } \\
\text { Stokes, 1997b }\end{array}$ & $\begin{array}{l}\text { Bright red } \\
\text { light in the } \\
\text { early or late } \\
\text { part of the } \\
\text { rearing period } \\
\text { (exp. } 2 \text { ) }\end{array}$ & - & $\begin{array}{l}\text { Bright red } \\
\text { light increased } \\
\text { activity in the } \\
\text { early part of } \\
\text { the rearing }\end{array}$ & $\begin{array}{l}\text { Bright red } \\
\text { light } \\
\text { increased } \\
\text { growth in } \\
\text { the early } \\
\text { part of the } \\
\text { rearing }\end{array}$ & $\begin{array}{l}\text { Reduced by } \\
\text { both early } \\
\text { and late } \\
\text { bright red } \\
\text { light }\end{array}$ & - & Ross & N/A \\
\hline
\end{tabular}




\begin{tabular}{|c|c|c|c|c|c|c|c|c|}
\hline \multirow[t]{2}{*}{ Reference } & \multirow[t]{2}{*}{ Treatment } & \multirow{2}{*}{$\begin{array}{l}\text { Preference } \\
\text { (if tested) }\end{array}$} & \multicolumn{3}{|c|}{ Effect on } & \multicolumn{2}{|c|}{ Genotype } & \multirow{2}{*}{$\begin{array}{l}\text { Stocking } \\
\text { density }\end{array}$} \\
\hline & & & $\begin{array}{l}\text { Behaviour and } \\
\text { welfare }\end{array}$ & Growth & $\begin{array}{l}\text { Locomotion } \\
\text { disorders }\end{array}$ & $\begin{array}{l}\text { Slow- } \\
\text { growing }\end{array}$ & $\begin{array}{l}\text { Fast- } \\
\text { growing }\end{array}$ & \\
\hline $\begin{array}{l}\text { De Santana } \\
\text { Eich, et al., } \\
2016\end{array}$ & $\begin{array}{l}\text { Blue and red } \\
\text { LED light until } \\
42 \text { days }\end{array}$ & - & $\begin{array}{l}\text { Birds exposed } \\
\text { to red LED } \\
\text { light were } \\
\text { more active } \\
\text { than birds } \\
\text { exposed to } \\
\text { blue LED light }\end{array}$ & - & - & - & $\begin{array}{l}\text { Cobb } \\
500\end{array}$ & $\begin{array}{l}12 \\
\text { birds/m2 }\end{array}$ \\
\hline $\begin{array}{l}\text { Asih, et al., } \\
2018\end{array}$ & $\begin{array}{l}\text { Intermittent } \\
\text { blue lighting } \\
\text { (IBL) and } \\
\text { continuous } \\
\text { blue lighting } \\
\text { (CBL) until } 28 \\
\text { days }\end{array}$ & - & $\begin{array}{l}\text { - Increased } \\
\text { duration and } \\
\text { reduced } \\
\text { frequency of } \\
\text { feeding in } \mathrm{CBL} \\
\text { - Reduced } \\
\text { corticosterone } \\
\text { hormone } \\
\text { concentration } \\
\text { in } \mathrm{CBL}\end{array}$ & $\begin{array}{l}\text { Increased } \\
\text { growth } \\
\text { rate in } \\
\mathrm{CBL}\end{array}$ & - & $\begin{array}{l}\text { Lohmann } \\
\text { (slow?) }\end{array}$ & - & $\mathrm{N} / \mathrm{A}$ \\
\hline $\begin{array}{l}\text { Khaliq, et al., } \\
2018\end{array}$ & $\begin{array}{l}\text { Blue, green } \\
\text { and red light } \\
\text { in the evening } \\
\text { hours until } 6 \\
\text { weeks }\end{array}$ & - & $\begin{array}{l}\text { - Increased } \\
\text { occurrence of } \\
\text { resting and } \\
\text { comfort } \\
\text { behaviours in } \\
\text { blue and } \\
\text { green light } \\
\text { - Increased } \\
\text { occurrence of } \\
\text { aggressive } \\
\text { behaviours in } \\
\text { red light }\end{array}$ & - & - & $\mathrm{N} / \mathrm{A}$ & $\mathrm{N} / \mathrm{A}$ & $\mathrm{N} / \mathrm{A}$ \\
\hline
\end{tabular}

As shown in table 10, little is known on the preference of broiler chickens for certain light colours. It seems that broilers show a preference towards red colour light (Senaratna, et al., 2018), which may be explained by the increased levels of activity found when birds are exposed to red light at different periods or throughout the entire rearing period. Nevertheless, colour preference tests have been hardly applied in experiments where lighting parameters were investigated in broilers. Next to that, setting of an optimum light intensity is difficult since dim intensities that favour growth reduce welfare (Senaratna, et al., 2018). Thus, studies where colour preferences are tested are needed, and when possible, also in combination with the testing of most effective intensity schedules that favours both performance and welfare in broiler chickens. 


\section{Discussion and conclusions}

The aim of the current report was to summarise the state-of-the art of knowledge with respect to effective environmental enrichment and lighting conditions for broiler chickens, as a starting point to define research questions specifically targeted to optimise housing conditions for slow-growing broiler chickens. This to better meet their behavioural needs and to optimise their welfare in commercial systems, while at the same time being practically applicable and economically efficient. Therefore, this literature study and practical inventory will form the basis for future trials regarding environmental enrichment and lighting conditions applied to slow-growing broiler production systems.

\subsection{Environmental enrichment}

Perching is considered part of the natural behaviour of chickens (Norring, et al., 2016). Therefore, elevated resting places (such as perches and platforms) are generally accepted as an effective form of enrichment for broiler chickens, particularly for fast-growing broiler chickens due to, for instance, positive effects found on leg health (Riber, et al., 2018). Nevertheless, in practice, the use of elevated resting places is still considered to be limited in most slow-growing broiler production systems used in the Netherlands at the moment. Moreover, from the literature study, perch use seems to be quite variable whereas platforms are generally well used by slow-growing broiler chickens. It should be mentioned, though, that this information originates from a limited number of scientific publications reporting on the use of elevated resting places by slow-growing broiler chickens in recent years (see Table 1). Sometimes perch use in slow-growing breeds is low, but this might be due to perch design, including aspects such as material used (e.g. wood, metal), shape of perches (e.g. oval, round, rounded upper edges), how these are provided (e.g. A-frame, with/without access ramp), height and dimensions. Potentially, perches may improve leg health in slow-growing broiler chickens as well. But there might be a risk of increased prevalence of breast blisters and keel bone damage with perches (Nielsen, 2004). Further research on perch use, and its effect on behaviour and other welfare indicators in slow growing breeds is needed. Evaluation of optimal designs of perches should consider possible positive or detrimental effects on bird health.

In the particular case of platforms, recent studies have indicated that they may reduce fearfulness and thereby improve welfare of fast-growing broiler chickens (Baxter, et al., 2019; Tahamtani, et al., 2018). So far, the of platforms effect on fearfulness has not been tested in slow-growing breeds, but it is an important aspect for these breeds due to their higher activity level than fast growing breeds (Bokkers and Koene, 2003; Rothschild, et al., 2019) as compared to fast-growing breeds. Slowgrowing broiler chickens may be very flighty and thereby damage each other, especially at catching. The provision of a more diverse environment, with for instance platforms combined with other type of enrichment (e.g. bales), may aid to improve leg health (Baxter, et al., 2018b; Vasdal, et al., 2019) and reduce smothering and injuries at catching by reducing fearfulness already during the production cycle.

Also, for both perches and platforms, it is interesting to test how large and/or how many of these elevated resting places should be provided in accordance to differences in group size and stocking densities encountered in the slow-growing broiler production systems used in the Netherlands. Is, for example, 2 meters of perches or 1 bale per 1000 birds, as applied in some production concepts, sufficient for during the whole production cycle?

With regard to other types of enrichment, from the literature study, the majority of the scientific publications (still mostly on fast-growing breeds) reported that bales of substrate (straw, lucerne hay or wood-shavings) are generally well used by both fast and slow-growing broiler chickens.

Nevertheless, the number of publications reporting on the use of bales by slow-growing breeds was very limited (Bergmann, et al., 2017; De Jong and Van Wijhe-Kiezebrink, 2014). So, it merits further 
research in slow-growing breeds. In addition, bales are one of the few types of enrichment with multiple functions that have not been fully explored in broiler production systems yet. This is because bales are used not only for perching, but also for clustering around it (protection), and when partly open (thus, not wrapped in plastic), birds may use it also for explorative behaviour (pecking). To date, no studies have specifically documented the multiple uses of one enrichment, which could be considered as providing for multiple behavioural needs of broilers. Also for bales, it is interesting to test if there is an optimum number of enrichments per number of chickens that should be provided, and for the purpose of stimulating explorative behaviour, different levels of loosely pressed bales should be tested against the compact, tightly pressed bales that are currently used in most slowgrowing broiler production systems used in the Netherlands at the moment. It is expected that loosely pressed bales will stimulate explorative behaviour more than tightly pressed bales, and possibly also at a younger age. So, loosely pressed bales could be provided to the birds already at the start of the production cycle, and different levels of looseness or firmness could be gradually introduced to the birds in accordance to their level of development and age.

Moreover, an important aspect of enrichment aimed at stimulating foraging and pecking behaviour is that it should remain interesting to the birds throughout the entire production cycle. This is difficult to maintain with non-food based pecking objects (e.g. strings, chains), but on the other hand, foodbased enrichment, such as larvae, have been shown to stimulate foraging behaviour in slow-growing broilers in a sustained manner during the entire production cycle (Blaauw, 2019). This is a type of enrichment that is hardly used in slow-growing broiler production systems in the Netherlands, which also merits further research ${ }^{2}$.

\subsection{Lighting conditions}

Currently, little is known about the need for light in slow-growing broiler chickens and how this interacts with the environmental enrichment offered. More insight in the specific requirements of slowgrowing broiler chickens with respect to light helps to provide housing conditions that better meet the needs of the birds, and thus contribute to broiler chickens' welfare. In consultation with stakeholders, it was decided to limit the literature study to three potential interesting areas of research: (1) effects of natural light provision and its variation across the broiler house; (2) effects of ultraviolet wavelengths on chicken behaviour; and (3) light colour preferences of broiler chickens.

From a few studies investigating the effect of natural light in combination with enrichment on chicken behaviour and welfare, there are indications that provision of natural light improves activity levels and leg health of fast-growing indoor-housed broiler chickens (De Jong and Gunnink, 2019; Bailie, et al., 2013). Moreover, dependent on weather conditions, natural light provides variation in light intensity across the broiler house, which may be relevant for promoting chicken welfare due to increased opportunity to perform different behaviours in different light environments. So, in farms where windows are provided along the side walls, resting behaviour occur more often in the darker areas (at lower light intensities) whereas active behaviours occur in the lighter areas (at higher light intensities), but it is up to the birds to choose under which circumstances they want to stay. In this way, provision of natural light to slow-growing breeds may be optimised, but this needs further research, where for instance, roof and side wall windows are compared.

Because natural light and enrichments may interact, the positioning of the enrichments in accordance to the variation in light intensity across the broiler house is also important. It would be interesting to test what would happen when birds are given the opportunity to choose between, for instance, a platform placed in a lighter area (likely stimulating activity) versus a platform placed in a darker area (likely stimulating resting). This may lead to the creation of different functional areas in the broiler house, and stimulate multiple uses of one single type of enrichment.

\footnotetext{
2 The Dutch SPA stresses the fact that there is insufficient knowledge on insect welfare when these are reared to be used as feed for chickens, and they therefore do currently not support this type of enrichment for broiler chickens.
} 
With regard to artificial light, light exposure during incubation may have positive effects on chicken welfare, by for instance, reducing fearfulness in later life (Archer and Mench, 2013; Archer and Mench, 2014). Moreover, light conditions at the hatchery or the breeder may already determine the colour preferences and affect behaviour of the offspring. But still, little is known on the preference of broiler chickens for certain light colours. So far, there are no studies that have been conducted in the Netherlands to test colour preferences in slow-growing breeds. Thus, studies on colour preferences in slow-growing broilers in relation to age and behaviour are directly needed. Also, in combination with variable colour temperature (cold and warm).

UV enrichment may have positive effects on behaviour, but studies on slow-growing breeds are not available at the moment. UVA wavelengths are visible to chickens and affects their perception of conspecifics (possibility of increasing visible plumage patterns in coloured birds) and their environment. So, it may be an effective and low-cost way to attract birds to enrichments, which might be more easy for some farmers to install.

In addition to light, provision of dark areas would be interesting, especially for young animals. So, studies on the provision of dark brooders to slow-growing breeds at the start of the production cycle are also encouraged.

\subsection{Recommendations for future research}

Regarding the current lack of knowledge on the behavioural requirements of slow-growing broiler chickens, various questions were raised, such as:

- What is the optimum perch design for slow-growing breeds and how does it affects (leg) health?

- What are the effects of certain types of enrichment, for instance platforms, on fearfulness?*

- Is there an optimum number of enrichments per number of chickens that should be provided?

- What is the effect of single enrichment with multiple functions compared to different enrichments on behaviour and welfare?*

- Do 'loosely pressed' bales meet the requirements for explorative behaviour (pecking) of the birds more than 'firmly pressed' bales?*

- Is it possible to use larvae as an attractive type of enrichment which can stimulate foraging behaviour even more?

- How does variation in light intensities across the broiler house affect behaviour and welfare of the birds?*

- Can we optimise light distribution across the broiler house by, for instance, providing light through windows along the side walls of the house instead of in the roof**

- Can we create different functional areas in the broiler house with different light intensities and/or colour temperatures in combination with enrichment?

- What are the colour preferences of slow-growing chickens in relation to age and behaviour?

- Can UV enrichment have positive effects on behaviour and welfare?

- Do young chicks avoid high intensity natural light? What are their preferences for natural light/intensity in relation to age? What is the effect of the provision of dark brooders to slowgrowing breeds at the start of the production cycle?*

- What are the effects of the interaction between lighting conditions and enrichment on chicken behaviour and welfare?

From this list, a limited number of research questions will be selected and used for the design of experiments on enrichment provision (year: 2020) and on light provision (year: 2021) in slow-growing broiler chickens. Priority will be given to the questions marked with an asterisk (some can be combined in one experiment). Different slow-growing breeds are used nowadays, and may differ with regard to their requirements for enrichment and light. Nevertheless, the main focus of the experiments will be on Hubbard breeds, which are considered representative of the Dutch slowgrowing broiler market at the moment. Although it is not in the scope of the project, epigenetic effects may be taken into account in one of the experiments, but a final decision will be made on the course of the project. 


\section{References}

Aksit, M., Z. K. Yardim, and S. Yalcin. 2017. Environmental enrichment influences on broiler performance and meat quality: Effect of light source and providing perches. European Poultry Science 81:10. doi 10.1399/eps.2017.182

Altan, O., C. Seremet, and H. Bayraktar. 2013. The effects of early environmental enrichment on performance, fear and physiological responses to acute stress of broiler. Arch. Geflugelkd. 77:23-28.

Alvino, G. M., R. A. Blatchford, G. S. Archer, and J. A. Mench. 2009. Light intensity during rearing affects the behavioural synchrony and resting patterns of broiler chickens. Br. Poult. Sci. 50:275-283. doi 10.1080/00071660902942775

Archer, G. S. 2017. Exposing broiler eggs to green, red and white light during incubation. Animal 11:12031209. doi $10.1017 / \mathrm{s} 1751731117000143$

Archer, G. S. 2018. Color temperature of light-emitting diode lighting matters for optimum growth and welfare of broiler chickens. Animal 12:1015-1021. doi 10.1017/s1751731117002361

Archer, G. S., and J. A. Mench. 2013. The effects of light stimulation during incubation on indicators of stress susceptibility in broilers. Poult. Sci. 92:3103-3108. doi 10.3382/ps.2013-03434

Archer, G. S., and J. A. Mench. 2014. Natural incubation patterns and the effects of exposing eggs to light at various times during incubation on post-hatch fear and stress responses in broiler (meat) chickens. Applied Animal Behaviour Science 152:44-51. doi 10.1016/j.applanim.2013.12.010

Archer, G. S., H. L. Shivaprasad, and J. A. Mench. 2009. Effect of providing light during incubation on the health, productivity, and behavior of broiler chickens. Poult. Sci. 88:29-37. doi 10.3382/ps.2008-00221

Arnould, C., D. Bizeray, J. M. Faure, and C. Leterrier. 2004. Effects of the addition of sand and string to pens on use of space, activity, tarsal angulations and bone composition in broiler chickens. Anim. Welf. 13:87-94.

Asih, D. R., S. Harimurti, and Wihandoyo. 2018. The effect of 12 and 24-hour blue lighting on performance and feeding behaviour of broiler chickens. Buletin Peternakan 42:15-19.

Avined, 2019. Antibioticumgebruik pluimveesector in 2018 en de trends van afgelopen jaren. AVINED, Nieuwegein, The Netherlands.

Bailie, C. L., M. E. E. Ball, and N. E. O'Connell. 2013. Influence of the provision of natural light and straw bales on activity levels and leg health in commercial broiler chickens. Animal 7:618-626. doi $10.1017 / \mathrm{s} 1751731112002108$

Bailie, C. L., M. Baxter, and N. E. O'Connell. 2018a. Exploring perch provision options for commercial broiler chickens. Applied Animal Behaviour Science 200:114-122. doi 10.1016/j.applanim.2017.12.007

Bailie, C. L., C. Ijichi, and N. E. O'Connell. 2018b. Effects of stocking density and string provision on welfarerelated measures in commercial broiler chickens in windowed houses. Poult. Sci. 97:1503-1510. doi $10.3382 / \mathrm{ps} /$ pey026

Bailie, C. L., and N. E. O'Connell. 2014. The effect of level of straw bale provision on the behaviour and leg health of commercial broiler chickens. Animal 8:1715-1721. doi 10.1017/s1751731114001529

Bailie, C. L., and N. E. O'Connell. 2015. The influence of providing perches and string on activity levels, fearfulness and leg health in commercial broiler chickens. Animal 9:660-668. doi $10.1017 / \mathrm{s} 1751731114002821$

Bailie, C. L., and N. E. O'Connell. 2016. Perch design preferences of commercial broiler chickens reared in windowed houses. Proc. EAAP 67th Annual Meeting, Belfast, Ireland.

Baxter, M., C. L. Bailie, and N. E. O'Connell. 2018a. An evaluation of potential dustbathing substrates for commercial broiler chickens. Animal 12:1933-1941. doi 10.1017/s1751731117003408

Baxter, M., C. L. Bailie, and N. E. O'Connell. 2018b. Evalution of dustbathing substrate and straw bales as environmental enrichments in commercial broiler housing. Applied Animal Behaviour Science 200:78-85. doi 10.1016/j.applanim.2017.11.010

Baxter, M., C. L. Bailie, and N. E. O'Connell. 2019. Play behaviour, fear responses and activity levels in commercial broiler chickens provided with preferred environmental enrichments. Animal 13:171-179. doi $10.1017 /$ s1751731118001118 
Baxter, M., and N. E. O'Connell. 2019. Does grouping environmental enrichments together affect the way they are used by commercially housed broiler chickens? Applied Animal Behaviour Science 210:52-59. doi 10.1016/j.applanim.2018.10.017

Baxter, M., and N. E. O'Connell. 2016. Commercial comparison of potential dustbathing substrates for intensively farmed broilers. Proc. EAAP 67th Annual Meeting, Belfast, Ireland.

Bench, C. J., M. A. Oryschak, D. R. Korver, and E. Beltranena. 2017. Behaviour, growth performance, foot pad quality, bone density, and carcass traits of broiler chickens reared with barrier perches and fed different dietary crude protein levels. Can. J. Anim. Sci. 97:268-280. doi 10.1139/cjas-2015-0202

Berghout, J., W. Roland, M. Vollebregt, M. Koene, and I. d. Jong. 2018. Towards a safe and sustainable poultry production chain. Wageningen Livestock Research, Wageningen, Report 1126.

Bergmann, S., A. Schwarzer, K. Wilutzky, H. Louton, J. Bachmeier, P. Schmidt, M. Erhard, and E. Rauch. 2017. Behavior as welfare indicator for the rearing of broilers in an enriched husbandry environmentd-A field study. Journal of Veterinary Behavior-Clinical Applications and Research 19:90-101. doi 10.1016/j.jveb.2017.03.003

Bizeray, D., I. Estevez, C. Leterrier, and J. M. Faure. 2002a. Effects of increasing environmental complexity on the physical activity of broiler chickens. Applied Animal Behaviour Science 79:27-41. doi 10.1016/s0168-1591(02)00083-7

Bizeray, D., I. Estevez, C. Leterrier, and J. M. Faure. 2002b. Influence of increased environmental complexity on leg condition, performance, and level of fearfulness in broilers. Poult. Sci. 81:767-773. doi $10.1093 / \mathrm{ps} / 81.6 .767$

Blaauw, X. E. 2019. Effects of pen enrichment on performance, walking ability and behaviour of fast and slow growing broilers. Master. Wageningen University and Research, Wageningen.

Blatchford, R. A., G. S. Archer, and J. A. Mench. 2012. Contrast in light intensity, rather than day length, influences the behavior and health of broiler chickens. Poult. Sci. 91:1768-1774. doi 10.3382/ps.201102051

Boissy, A., G. Manteuffel, M. B. Jensen, R. O. Moe, B. Spruijt, L. J. Keeling, C. Winckler, B. Forkman, I. Dimitrov, J. Langbein, M. Bakken, I. Veissier, and A. Aubert. 2007. Assessment of positive emotions in animals to improve their welfare. Physiol. Behav. 92:375-397. doi 10.1016/j.physbeh.2007.02.003

Bokkers, E. A. M., and P. Koene. 2003. Behaviour of fast- and slow growing broilers to 12 weeks of age and the physical consequences. Applied Animal Behaviour Science 81:59-72. doi https://doi.org/10.1016/S0168-1591(02)00251-4

Cornetto, T., and I. Estevez. 2001a. Behavior of the domestic fowl in the presence of vertical panels. Poult. Sci. 80:1455-1462. doi $10.1093 / \mathrm{ps} / 80.10 .1455$

Cornetto, T., and I. Estevez. 2001b. Influence of vertical panels on use of space by domestic fowl. Applied Animal Behaviour Science 71:141-153. doi 10.1016/s0168-1591(00)00171-4

Cornetto, T., I. Estevez, and L. W. Douglass. 2002. Using artificial cover to reduce aggression and disturbances in domestic fowl. Applied Animal Behaviour Science 75:325-336. doi 10.1016/s01681591(01)00195-2

Dawkins, M. S. 1989. Time budgets in red junglefowl as a baseline for the assessment of welfare in domestic fowl. App. Anim. Behav. Sci. 24:77-80.

De Jong, I. C., and H. Gunnink. 2019. Effects of a commercial broiler enrichment programme with or without natural light on behaviour and other welfare indicators. Animal 13:384-391. doi $10.1017 / \mathrm{s} 1751731118001805$

De Jong, I. C., and M. Göertz. 2017. Broiler chicken stocking density affects use of environmental enrichment objects. Proc. Xth European Symposium on Poultry Welfare, Ploufragan, France.

De Jong, I. C., and M. C. Van Wijhe-Kiezebrink. 2014. Use of different types of enrichment in slower growing broilers; Wageningen Livestock Research, Wageningen, Report 810.

de Santana Eich, M. R., R. Garcia, I. Nääs, F. Caldara, R. Borille, A. Flavia Basso Roye, and S. Sgavioli. 2016. Behavior of Broilers Reared under Monochromatic and Fluorescent Light Sources. International Journal of Poultry Science 15:96-102. doi 10.3923/ijps.2016.96.102

Dierenbescherming, 2019. Vleeskuikenconcepten in Nederland. Een vergelijking op gebied van dierenwelzijn. EFSA. 2010. Scientific Opinion on the influence of genetic parameters on the welfare and the resistance to stress of commercial broilers. EFSA journal 8:1666

EFSA. 2015. Scientific Opinion on welfare aspects of the use of perches for laying hens. EFSA Journal 13:4131. doi 10.2903/j.efsa.2015.4131 
Ellen, H. H., F. R. Leenstra, R. A. van Emous, C. M. Groenestein, J. van Harn, P. L. M. van Horne, I. C. de Jong, M. Kense, D. J. Mevius, and J. A. Wagenaar. 2012. Vleeskuikenproductiesystemen in Nederland (Broiler production systems in The Netherlands). Wageningen Livestock Research, Lelystad, Report 619.

Estevez, I., N. Tablante, R. L. Pettit-Riley, and L. Carr. 2002. Use of cool perches by broiler chickens. Poult. Sci. 81:62-69. doi 10.1093/ps/81.1.62

Fraser, D. 1995. Science, values and animal-welfare - exploring the inextricable connection. Anim. Welf. 4:103-117.

Groves, P. J., and W. I. Muir. 2013. Use of perches by broiler chickens in floor pen experiments. Proc. 9th European Symposium on Poultry Welfare, Uppsala, Sweden.

Hesham, M. H., A. H. El Shereen, and S. N. Enas. 2018. Impact of different light colors in behavior, welfare parameters and growth performance of Fayoumi broiler chickens strain. J. Hell. Vet. Med. Soc. 69:951958. doi 10.12681/jhvms.18017

Huth, J. C., and G. S. Archer. 2015. Comparison of Two LED Light Bulbs to a Dimmable CFL and their Effects on Broiler Chicken Growth, Stress, and Fear. Poult. Sci. 94:2027-2036. doi 10.3382/ps/pev215

James, C., L. Asher, K. Herborn, and J. Wiseman. 2018. The effect of supplementary ultraviolet wavelengths on broiler chicken welfare indicators. Applied Animal Behaviour Science 209:55-64. doi 10.1016/j.applanim.2018.10.002

Jordan, D., I. Stuhec, and W. Bessei. 2011. Effect of whole wheat and feed pellets distribution in the litter on broilers' activity and performance. Arch. Geflugelkd. 75:98-103.

Kaukonen, E., M. Norring, and A. Valros. 2016. Using elevated platforms to improve broiler leg health on commercial broiler farms. Proc. 50th Congress of the International Society of Applied Ethology, Edinburgh, UK.

Kaukonen, E., M. Norring, and A. Valros. 2017. Perches and elevated platforms in commercial broiler farms: use and effect on walking ability, incidence of tibial dyschondroplasia and bone mineral content. Animal 11:864-871. doi 10.1017/s1751731116002160

Kells, A., M. S. Dawkins, and M. C. Borja. 2001. The effect of a 'freedom food' enrichment on the behaviour of broilers on commercial farms. Anim. Welf. 10:347-356.

Khaliq, T., A. Khan, T. Nazir, and B. Mir. 2018. Behavior study of broilers reared under different colors of light in the evening hours. Journal of Entomology and Zoology Studies 6:1624-1627.

Kristensen, H. H., N. B. Prescott, G. C. Perry, J. Ladewig, A. K. Ersboll, K. C. Overvad, and C. M. Wathes. 2007. The behaviour of broiler chickens in different light sources and illuminances. Applied Animal Behaviour Science 103:75-89. doi 10.1016/j.applanim.2006.04.017

LeVan, N. F., I. Estevez, and W. R. Stricklin. 2000. Use of horizontal and angled perches by broiler chickens. Applied Animal Behaviour Science 65:349-365. doi 10.1016/s0168-1591(99)00059-3

Maddocks, S. A., I. C. Cuthill, A. R. Goldsmith, and C. M. Sherwin. 2001. Behavioural and physiological effects of absence of ultraviolet wavelengths for domestic chicks. Anim. Behav. 62:1013-1019. doi 10.1006/anbe.2001.1842

Malchow, J., B. Puppe, J. Berk, and L. Schrader. 2019. Effects of Elevated Grids on Growing Male Chickens Differing in Growth Performance. Frontiers in Veterinary Science 6. doi 10.3389/fvets.2019.00203

Marino, L. 2017. Thinking chickens: a review of cognition, emotion, and behavior in the domestic chicken. Anim. Cogn. 20:127-147. doi 10.1007/s10071-016-1064-4

Martrenchar, A., D. Huonnic, J. P. Cotte, E. Boilletot, and J. P. Morisse. 2000. Influence of stocking density, artificial dusk and group size on the perching behaviour of broilers. Br. Poult. Sci. 41:125-130. doi $10.1080 / 713654921$

Mendes, A. S., S. J. Paixão, R. Restelatto, G. M. Morello, D. Jorge de Moura, and J. C. Possenti. 2013. Performance and preference of broiler chickens exposed to different lighting sources. The Journal of Applied Poultry Research 22:62-70. doi 10.3382/japr.2012-00580

Niekerk, T. v., H. Ellen, and A. Winkel. 2015. Licht op licht : licht en verlichting in de pluimveehouderij in relatie tot beschadigend pikgedrag. Wageningen Livestock Research, Wageningen, Report 922.

Nielsen, B. L. 2004. Breast blisters in groups of slow-growing broilers in relation to strain and the availability and use of perches. Br. Poult. Sci. 45:306-315. doi 10.1080/00071660410001730798

Norring, M., E. Kaukonen, and A. Valros. 2016. The use of perches and platforms by broiler chickens. Applied Animal Behaviour Science 184:91-96. doi 10.1016/j.applanim.2016.07.012

Pedersen, I. J., F. M. Tahamtani, and A. B. Riber. 2017. Effects of elevated platforms on fearfulness in fastgrowing broilers. Proc. 51st Congress of the International Society of Applied Ethology, Aarhus, Denmark.

Pettit-Riley, R., and I. Estevez. 2001. Effects of density on perching behavior of broiler chickens. Applied Animal Behaviour Science 71:127-140. doi 10.1016/s0168-1591(00)00174-x 
Pettit-Riley, R., I. Estevez, and E. Russek-Cohen. 2002. Effects of crowding and access to perches on aggressive behaviour in broilers. Applied Animal Behaviour Science 79:11-25. doi 10.1016/s01681591(02)00116-8

Pichova, K., J. Nordgreen, C. Leterrier, L. Kostal, and R. O. Moe. 2016. The effects of food-related environmental complexity on litter directed behaviour, fear and exploration of novel stimuli in young broiler chickens. Applied Animal Behaviour Science 174:83-89. doi 10.1016/j.applanim.2015.11.007

Prayitno, D., C. Phillips, and H. Omed. 1997a. The effects of color of lighting on the behavior and production of meat chickens. Poult. Sci. 76:452-457. doi 10.1093/ps/76.3.452

Prayitno, D., C. Phillips, and D. Stokes. 1997b. The effects of color and intensity of light on behavior and leg disorders in broiler chickens. Poult. Sci. 76:1674-1681. doi 10.1093/ps/76.12.1674

Prescott, N. B., and C. M. Wathes. 1999. Spectral sensitivity of the domestic fowl (Gallus g. domesticus). Br. Poult. Sci. 40:332-339. doi 10.1080/00071669987412

Rault, J. L., K. Clark, P. J. Groves, and G. M. Cronin. 2017. Light intensity of 5 or 20 lux on broiler behavior, welfare and productivity. Poult. Sci. 96:779-787. doi 10.3382/ps/pew423

Riber, A. B. 2015. Effects of color of light on preferences, performance, and welfare in broilers. Poult. Sci. 94:1767-1775. doi 10.3382/ps/pev174

Riber, A. B., H. A. van de Weerd, I. C. de Jong, and S. Steenfeldt. 2018. Review of environmental enrichment for broiler chickens. Poult. Sci. 97:378-396. doi 10.3382/ps/pex344

Rodriguez-Aurrekoetxea, A., E. H. Leone, and I. Estevez. 2014. Environmental complexity and use of space in slow growing free range chickens. Applied Animal Behaviour Science 161:86-94. doi 10.1016/j.applanim.2014.09.014

Rodriguez-Aurrekoetxea, A., E. H. Leone, and I. Estevez. 2015. Effects of panels and perches on the behaviour of commercial slow-growing free-range meat chickens. Applied Animal Behaviour Science 165:103-111. doi 10.1016/j.applanim.2015.02.004

Rothschild, D., M. Nascimento dos Santos, T. M. Widowski, N. A. Karrow, L. Susta, E. Kiarie, I. Mandell, and S. Torrey. 2019. A comparison of organ size between conventional and slower growing broiler chickens. Proc. Poultry Science Association Annual Meeting, Montréal, Québec, Canada.

Ruis, M. A. W., E. Coenen, J. v. Harn, P. Lenskens, and T. B. Rodenburg. 2004. Effect of an outdoor run and natural light on welfare of fast growing broilers. Proc. 38th Congress of the International Society of Applied Ethology, Helsinki, Finland.

Ruis, M. A. W., B. F. J. Reuvekamp, H. Gunnink, and G. P. Binnendijk. 2010. The effect of optimized lighting conditions on feather pecking and production of laying hens. Wageningen Livestock Research, Lelystad, Report 335.

Saatkamp, H. W., L. S. M. Vissers, P. L. M. van Horne, and I. C. de Jong. 2019. Transition from conventional broiler meat to meat from production concepts with higher animal welfare: Experiences from The Netherlands. Animals 9:483.

Sandilands, V., C. Moinard, and N. H. C. Sparks. 2009. Providing laying hens with perches: fulfilling behavioural needs but causing injury? Br. Poult. Sci. 50:395-406. doi 10.1080/00071660903110844

Senaratna, D., T. S. Samarakone, and W. W. D. A. Gunawardena. 2018. Effects of four dim vs high intensity red color light regimens on growth performance and welfare of broilers. Asian Australas. J. Anim. Sci. 31:149-156. doi 10.5713/ajas.16.0139

Su, G., P. Sorensen, and S. C. Kestin. 2000. A note on the effects of perches and litter substrate on leg weakness in broiler chickens. Poult. Sci. 79:1259-1263. doi 10.1093/ps/79.9.1259

Tahamtani, F. M., I. J. Pedersen, C. Toinon, and A. B. Riber. 2018. Effects of environmental complexity on fearfulness and learning ability in fast growing broiler chickens. Applied Animal Behaviour Science 207:49-56. doi https://doi.org/10.1016/j.applanim.2018.04.005

Vasdal, G., J. Vas, R. C. Newberry, and R. O. Moe. 2019. Effects of environmental enrichment on activity and lameness in commercial broiler production. Journal of Applied Animal Welfare Science 22:197-205. doi 10.1080/10888705.2018.1456339

Ventura, B. A., F. Siewerdt, and I. Estevez. 2010. Effects of barrier perches and density on broiler leg health, fear, and performance. Poult. Sci. 89:1574-1583. doi 10.3382/ps.2009-00576

Ventura, B. A., F. Siewerdt, and I. Estevez. 2012. Access to Barrier Perches Improves Behavior Repertoire in Broilers. PLoS One 7:7. doi 10.1371/journal.pone.0029826

Vissers, L. S. M., I. C. de Jong, P. L. M. van Horne, and H. W. Saatkamp. 2019. Global Prospects of the CostEfficiency of Broiler Welfare in Middle-Segment Production Systems. Animals 9:473.

Welfare Quality, 2009. Welfare Quality® Assessment protocol for poultry. Welfare Quality® Consortium, Lelystad, The Netherlands. 
Yildirim, M., and A. Taskin. 2017. The effects of Environmental Enrichment on Some Physiological and Behavioral Parameters of Broiler Chicks. Brazilian Journal of Poultry Science 19:355-362. doi 10.1590/1806-9061-2016-0402

Yngvesson, J., A. Wallenbeck, L. Jonsson, and S. Gunnarsson. 2016. Behaviour of broilers in semicommercial organic rearing - behaviour and mortality of hybrids with rapid or slow growth rate. Proc. 50th Congress of the International Society of Applied Ethology, Edinburgh, UK.

Zhao, J. P., H. C. Jiao, Y. B. Jiang, Z. G. Song, X. J. Wang, and H. Lin. 2013. Cool perches improve the growth performance and welfare status of broiler chickens reared at different stocking densities and high temperatures. Poult. Sci. 92:1962-1971. doi 10.3382/ps.2012-02933 


\section{Appendix 1}

Table 1 Overview of the requirements for the provision of daylight, dark period and use of enrichment in systems used for broiler production in The Netherlands (N/A = information not available). Source: Henk-Jan Schuurman (De Hoop Mengvoeders B.V., Zelhem, The Netherlands, personal communication); Ellen, et al. (2012); Saatkamp, et al. (2019), Dierenbescherming (2019).

\begin{tabular}{|c|c|c|c|c|c|}
\hline \multirow[t]{2}{*}{ Concept } & \multicolumn{3}{|c|}{ Requirements } & \multirow[t]{2}{*}{ Maximum daily growth ${ }^{1}$} & \multirow[t]{2}{*}{ Stocking density } \\
\hline & Daylight in the stable & Dark period & Enrichment & & \\
\hline Regular (EU legislation) & No requirement & $\begin{array}{l}6 \mathrm{~h} \text { of darkness, of which } 4 \mathrm{~h} \\
\text { are continuous }\end{array}$ & No requirement & Not regulation & $\begin{array}{l}18-21 \text { birds } / \mathrm{m} 2(33,39 \text { or } \\
\text { max. } 42 \mathrm{~kg} / \mathrm{m} 2)\end{array}$ \\
\hline McDonald's (regular) & $\begin{array}{l}\text { Provided through } \\
\text { openings in the roof or in } \\
\text { the facades (at least } 3 \% \\
\text { of the useable area) }\end{array}$ & N/A & $\begin{array}{l}\text { During the whole production } \\
\text { cycle: } 2 \text { meters of perches } \\
\text { per } 1000 \text { birds, and at least } \\
1 \text { pecking object per } 1000 \\
\text { birds. From } 8 \text { days of age } \\
\text { onwards: } 1.5 \text { bale of straw, } \\
\text { hay or Lucerne, or plastic } \\
\text { packaged wood-shavings (at } \\
\text { least } 10 \mathrm{~kg} \text { ) per } 1000 \text { birds }\end{array}$ & Not specified & $\mathrm{N} / \mathrm{A}$ \\
\hline $\begin{array}{l}\text { Deen, Deka, Dirk, COOP, } \\
\text { Hoogvliet, Jan Linders, } \\
\text { MCD, Poiesz, Spar, Vomar } \\
\text { (Groenland Kip) }\end{array}$ & $\begin{array}{l}\text { At least } 3 \% \text { of the } \\
\text { useable area }\end{array}$ & $6 \mathrm{~h}$ of continuous darkness & $\begin{array}{l}\text { Pecking stones ( } 1 \text { stone/250 } \\
\mathrm{m} 2) \text {, elevated platforms } \\
(1 \mathrm{~m} 2 / 2500 \text { birds) }\end{array}$ & 50 gram & $\begin{array}{l}15-16 \text { birds } / \mathrm{m} 2 \text { (max. } 38 \\
\mathrm{~kg} / \mathrm{m} 2 \text { ) }\end{array}$ \\
\hline $\begin{array}{l}\text { Boon's markt and MCD } \\
\text { (Betere kip), Picnic and } \\
\text { Boni (Comfort), } \\
\text { Nettorama (Kiplekker) }\end{array}$ & $\begin{array}{l}\text { At least } 3 \% \text { of the } \\
\text { useable area }\end{array}$ & $6 \mathrm{~h}$ of continuous darkness & $\begin{array}{l}\text { From } 15 \text { days of age } \\
\text { onwards: } 1 \text { bale of straw, } \\
\text { Lucerne or hay per } 500 \\
\text { birds, } 2 \text { grams of grain per } \\
\text { bird scattered on the ground }\end{array}$ & 50 gram & 15 birds/m2 \\
\hline
\end{tabular}




\begin{tabular}{|c|c|c|c|c|c|}
\hline \multirow[t]{2}{*}{ Concept } & \multicolumn{3}{|c|}{ Requirements } & \multirow[t]{2}{*}{ Maximum daily growth ${ }^{1}$} & \multirow[t]{2}{*}{ Stocking density } \\
\hline & Daylight in the stable & Dark period & Enrichment & & \\
\hline $\begin{array}{l}\text { Albert Heijn (nieuwe } A H \\
\text { kip) }\end{array}$ & No requirement & $6 \mathrm{~h}$ of continuous darkness & 1 straw bale/1000 birds & 50 gram & $\max .38 \mathrm{~kg} / \mathrm{m} 2$ \\
\hline Plus (nieuwe Plus kip) & $\begin{array}{l}\text { At least } 3 \% \text { of the } \\
\text { useable area }\end{array}$ & $6 \mathrm{~h}$ of darkness & 1 straw bale/1000 birds & 50 gram & $\max .38 \mathrm{~kg} / \mathrm{m} 2$ \\
\hline LIDL & $\begin{array}{l}\text { At least } 3 \% \text { of the } \\
\text { useable area }\end{array}$ & $6 \mathrm{~h}$ of continuous darkness & $\begin{array}{l}\text { Distraction material, such as } \\
\text { pecking objects ( } 1 \text { object } \\
\text { per } 150 \mathrm{~m}^{2} \text { of useable } \\
\text { area), and elevated } \\
\text { platforms ( } 1 \text { element per } \\
150 \mathrm{~m}^{2} \text { of useable area) }\end{array}$ & 50 gram & $\max .38 \mathrm{~kg} / \mathrm{m} 2$ \\
\hline Aldi & $\begin{array}{l}\text { At least } 3 \% \text { of the } \\
\text { useable area }\end{array}$ & $6 \mathrm{~h}$ of continuous darkness & $\begin{array}{l}\text { From } 15 \text { days of age } \\
\text { onwards: } 2 \text { grams of grain } \\
\text { per bird scattered on the } \\
\text { ground, } 1 \text { straw bale per } \\
1000 \text { birds or } 1 \text { pecking } \\
\text { stone per } 200 \mathrm{~m} 2\end{array}$ & 50 gram & 15 birds $/ \mathrm{m} 2(\max .34 \mathrm{~kg} / \mathrm{m} 2)$ \\
\hline $\begin{array}{l}\text { Jumbo (nieuwe standaard } \\
\text { kip) }\end{array}$ & $\begin{array}{l}\text { At least } 3 \% \text { of the } \\
\text { useable area }\end{array}$ & $8 \mathrm{~h}$ of continuous darkness & $\begin{array}{l}\text { From } 15 \text { days of age } \\
\text { onwards: daily scattering of } \\
\text { at least } 2 \text { grams of grain per } \\
\text { bird, } 1 \text { whole bale (at least } \\
20 \mathrm{~kg} \text { ) of straw, hay, } \\
\text { Lucerne or maize per } 1000 \\
\text { birds }\end{array}$ & 45 gram & $\begin{array}{l}13.5 \text { birds/m2 (max. } 30 \\
\mathrm{~kg} / \mathrm{m} 2)\end{array}$ \\
\hline $\begin{array}{l}\text { Beter Leven keurmerk ( } 1 \\
\text { star) }\end{array}$ & $\begin{array}{l}\text { At least } 3 \% \text { of the } \\
\text { useable area }\end{array}$ & $\begin{array}{l}\text { At least } 8 \text { h of continuous } \\
\text { darkness }\end{array}$ & $\begin{array}{l}\text { From } 8 \text { days of age } \\
\text { onwards: } 1 \text { bale }(15-20 \mathrm{~kg}) \\
\text { of straw, hay or Lucerne per } \\
1000 \text { birds. From } 15 \text { days of } \\
\text { age onwards: daily } \\
\text { scattering of at least } 2\end{array}$ & 45 gram & 12 birds $/ \mathrm{m} 2(\max .25 \mathrm{~kg} / \mathrm{m} 2)$ \\
\hline
\end{tabular}




\begin{tabular}{|c|c|c|c|c|c|}
\hline \multirow[t]{2}{*}{ Concept } & \multicolumn{3}{|c|}{ Requirements } & \multirow[t]{2}{*}{ Maximum daily growth ${ }^{1}$} & \multirow[t]{2}{*}{ Stocking density } \\
\hline & Daylight in the stable & Dark period & Enrichment & & \\
\hline & & & $\begin{array}{l}\text { grams of grain (or feed) per } \\
\text { bird, done manually by the } \\
\text { farmer }\end{array}$ & & \\
\hline Organic & Provided & $\begin{array}{l}\text { At least } 8 \mathrm{~h} \text { of continuous } \\
\text { darkness }\end{array}$ & No requirement & Not specified & 10 birds $/ \mathrm{m} 2(\max .21 \mathrm{~kg} / \mathrm{m} 2)$ \\
\hline
\end{tabular}

1 Dependent on the supermarket concept, different slow growing breeds may be used such as Hubbard JA (957, 757, 257), Ranger classic, Rowan Ranger, Ranger Gold, Rambler Ranger, Cobb Sasso 150 , Sasso SA 451 N. Hubbard breeds have a share of approximately $75 \%$ of the total Dutch slow-growing broiler market (Van Boekholt, pers. comm.). Slow-growing broiler chickens are defined as broiler chickens produced by slow-growing female parent stock that are crossed with a regular or slow-growing male. These broiler breeds are either accredited by the Dutch SPA with the 'Beter Leven' quality label (maximum average daily growth of 45 grams) or fall within the standards of the original 'Kip van Morgen' concept (maximum average daily growth of 50 grams) (Ellen, et al., 2012; Saatkamp, et al., 2019). 
Table 2 Overview of the requirements for the provision of daylight, dark period and use of enrichment in systems used for broiler production in the United Kingdom. Source: Annie Rayner (FAI Farms, Oxford, UK), pers. comm.

\begin{tabular}{|c|c|c|c|c|c|c|c|}
\hline \multirow[t]{2}{*}{ Concept } & \multirow[t]{2}{*}{ Retailer } & \multicolumn{3}{|c|}{ Requirements } & \multicolumn{2}{|c|}{ Genotype } & \multirow{2}{*}{$\begin{array}{l}\text { Stocking } \\
\text { density }\end{array}$} \\
\hline & & Daylight in the stable & Dark period & Enrichment & Slow-growing & Fast-growing & \\
\hline $\begin{array}{l}\text { UK Standard Red Tractor }{ }^{\mathrm{a}} \\
\text { Ingredients/added } \\
\text { value/Entry level }\end{array}$ & $\begin{array}{l}\text { TESCO, Sainbury's, } \\
\text { ASDA, Morrisons, } \\
\text { Co-op, ALDI, LIDL, } \\
\text { Waitrose, M\&S }\end{array}$ & $\begin{array}{l}\text { Evenly spaced and } \\
\text { minimum } 3 \% \\
\text { (recommended) floor } \\
\text { area in windows }\end{array}$ & $\begin{array}{l}\text { Minimum } 6 \text { hours, with } \\
1 \text { period of } 4 \text { hours } \\
\text { uninterrupted every } 24 \\
\text { hours }\end{array}$ & $\begin{array}{l}1 \text { bale, } 2 \mathrm{~m} \text { of perches } \\
\text { or } 0.3 \mathrm{~m}^{2} \text { platform or } 1 \\
\text { pecking object per } \\
1000 \text { birds }\end{array}$ & Not specified & Permitted & $38 \mathrm{~kg} / \mathrm{m}^{2}$ \\
\hline $\begin{array}{l}\text { UK Higher Welfare Retailer } \\
\text { Schemes }\end{array}$ & Waitrose and M\&S & $\begin{array}{l}\text { Evenly spaced and } \\
\text { minimum } 3 \% \text { floor area } \\
\text { in windows }\end{array}$ & $\begin{array}{l}\text { Minimum } 6 \text { hours, with } \\
1 \text { period of } 4 \text { hours } \\
\text { uninterrupted every } 24 \\
\text { hours }\end{array}$ & Provision of bales & Not specified & Cobb $(M \& S)$ & $30 \mathrm{~kg} / \mathrm{m}^{2}$ \\
\hline RSPCA Assured Indoor & Sainsbury's & $\begin{array}{l}\text { Minimum 3\% floor area } \\
\text { in windows }\end{array}$ & $6-12$ hours continuous & $\begin{array}{l}1.5 \text { bales, a } 2 \mathrm{~m} \text { perch } \\
\text { and } 1 \text { pecking object } \\
\text { per } 1000 \text { birds }\end{array}$ & $\begin{array}{l}\text { JA57, JA787, } \\
\text { JA987, Ranger } \\
\text { Classic, Ranger } \\
\text { Gold, Rambler } \\
\text { Ranger }\end{array}$ & Not permitted & $30 \mathrm{~kg} / \mathrm{m}^{2}$ \\
\hline Free range & $\begin{array}{l}\text { TESCO, Sainsbury's, } \\
\text { ASDA, Co-op, } \\
\text { Morrisons, ALDI, } \\
\text { LIDL, Waitrose, M\&S }\end{array}$ & $\begin{array}{l}\text { Natural light outdoors } \\
\text { and through pop holes }\end{array}$ & $\begin{array}{l}\text { Minimum } 6 \text { hours } \\
\text { continuous }\end{array}$ & $\begin{array}{l}1 \text { bale and } 1 \text { pecking } \\
\text { object per } 1000 \text { birds, } \\
2 \mathrm{~m} \text { of perches and } \\
0.3 \mathrm{~m}^{2} \text { platform per } 100 \\
\text { birds }\end{array}$ & Permitted & $\begin{array}{l}\text { Reared until } 56 \\
\text { days }\end{array}$ & $27.5 \mathrm{~kg} / \mathrm{m}^{2}$ \\
\hline Organic & $\begin{array}{l}\text { TESCO, Sainsbury's, } \\
\text { ASDA, Co-op, } \\
\text { Morrisons, M\&S, } \\
\text { Waitrose }\end{array}$ & Plentiful natural light & $\begin{array}{l}\text { Minimum } 8 \text { hours } \\
\text { continuous }\end{array}$ & $\begin{array}{l}\text { Perches, } \\
\text { straw/hay/alfalfa bales, } \\
\text { vegetables and other } \\
\text { vegetation, with at } \\
\text { least } 2 \text { enrichments per } \\
500 \text { chickens. }\end{array}$ & $\begin{array}{l}\text { Slower growing } \\
\text { recommended but } \\
\text { not mandatory }\end{array}$ & $\begin{array}{l}\text { Reared until } 70 \\
\text { days }\end{array}$ & $\begin{array}{l}21 \mathrm{~kg} / \mathrm{m}^{2} \\
\left(30 \mathrm{~kg} / \mathrm{m}^{2}\right. \\
\text { mobile } \\
\text { housing })\end{array}$ \\
\hline
\end{tabular}

aFrom $1^{\text {st }}$ October 2020. 


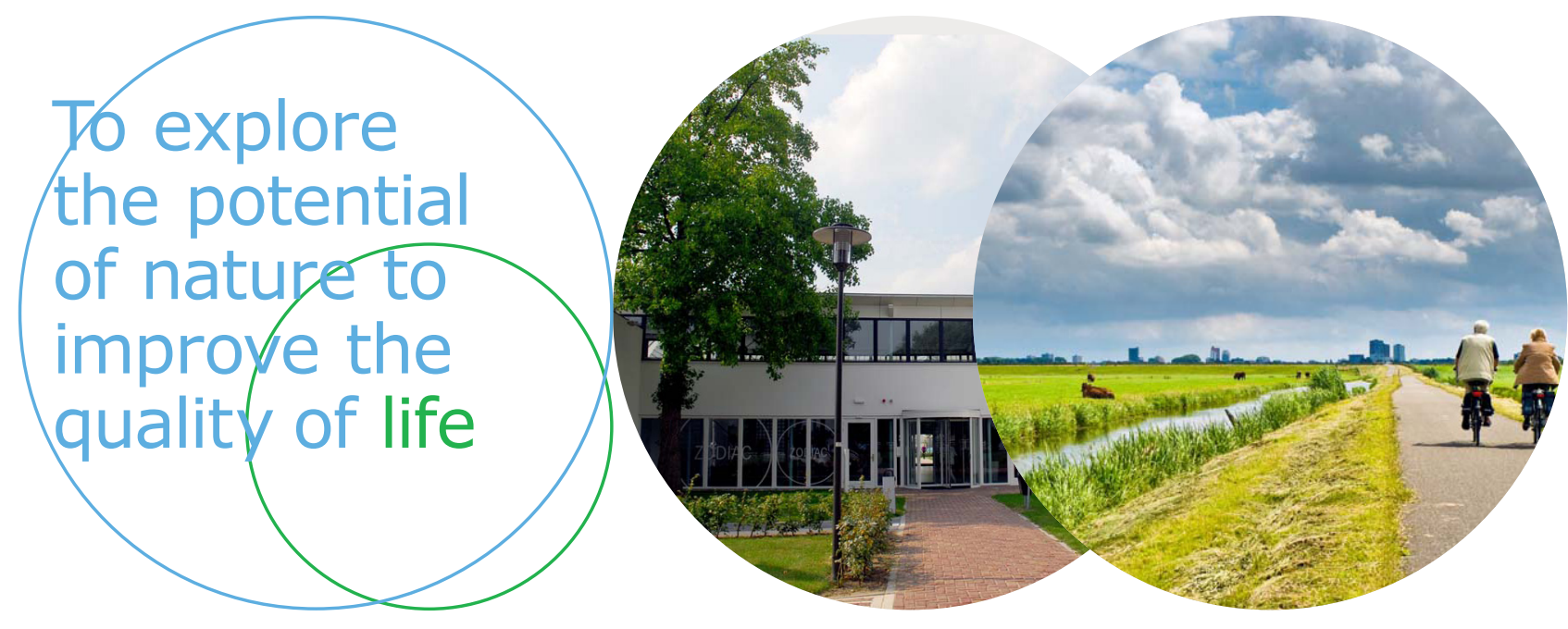

Wageningen Livestock Research P.O. Box 338

6700 AH Wageningen

The Netherlands

$\mathrm{T}+31(0) 317483953$

E info.livestockresearch@wur.nl

www.wur.nl/livestock-research

Wageningen Livestock Research creates science based solutions for a sustainable and profitable livestock sector. Together with our clients, we integrate scientific knowledge and practical experience to develop livestock concepts for future generations.

Wageningen Livestock Research is part of Wageningen University \& Research. Together we work on the mission: 'To explore the potential of nature to improve the quality of life'. A staff of 6,500 and 10,000 students from over 100 countries are working worldwide in the domain of healthy food and living environment for governments and the business community-at-large. The strength of Wageningen University \& Research lies in its ability to join the forces of specialised research institutes and the university. It also lies in the combined efforts of the various fields of natural and social sciences. This union of expertise leads to scientific breakthroughs that can quickly be put into practice and be incorporated into education. This is the Wageningen Approach. 